\title{
Torino 6 unica. Un'indagine preliminare sul manoscritto Varia 42/2 della Biblioteca Reale
}

\section{Alberto Rizzuti}

\section{(2) OpenEdition}

1 Journals

\section{Edizione digitale}

URL: https://journals.openedition.org/studifrancesi/22104

DOI: $10.4000 /$ studifrancesi.22104

ISSN: 2421-5856

\section{Editore}

Rosenberg \& Sellier

\section{Edizione cartacea}

Data di pubblicazione: 1 avril 2020

Paginazione: 84-112

ISSN: 0039-2944

\section{Notizia bibliografica digitale}

Alberto Rizzuti, «Torino 6 unica. Un'indagine preliminare sul manoscritto Varia 42/2 della Biblioteca

Reale», Studi Francesi [Online], 190 (LXIV | I) | 2020, online dal 01 avril 2021, consultato il 02 août 2021. URL: http://journals.openedition.org/studifrancesi/22104 ; DOI: https://doi.org/10.4000/studifrancesi. 22104

\section{(c) (i) (9)}

Studi Francesi è distribuita con Licenza Creative Commons Attribuzione - Non commerciale - Non opere derivate 4.0 Internazionale. 


\section{Torino 6 unica. \\ Un'indagine preliminare sul manoscritto Varia 42/2 della Biblioteca Reale}

\section{Abstract}

Turin's Royal Library preserves an early- $14^{\text {th }}$-century parchment manuscript containing three conducti and thirty-one motets. Six of its items (one conductus and five motets) are unica, yet their verbal texts have never been critically edited thus far. This article provides a full transcription and a comment of the texts, both in Latin and in French; moreover, it offers a preliminary survey on the entire manuscript, summing up the hypotheses surfaced along one century of episodic attention devoted to its content, before and after its only full transcription, published in 1953. The relationship to the two main collections of motets (mss. Bamberg and Montpellier) and to many manuscripts preserving trouvère songs (often with, sometimes without music) is one of the primary focuses; furthermore, the article explores the plurality of models represented in the small collection of conducti and in the large collection of motets, figuring out a possible relationship between them. While no conclusive statement can be made in the absence of a critical edition of all texts and music, some phenomena concerning the unica are highlighted: among these, use of Latin vs French Tenor, presence vs absence of a rhythmic mode in one or all parts, "Petronian" features of the Triplum, use of the same melody in the Tenor of a motet and in one of the upper voices in another, presence vs absence of spots showing voice-exchange (Stimmtausch), number and position of thirds in the resulting harmony.

La Biblioteca Reale di Torino conserva un manoscritto musicale pergamenaceo databile al secondo decennio del Trecento contenente tre conducti e trentuno mottetti, tutti a tre voci. Su trentaquattro composizioni, sei (un conductus e cinque mottetti) non risultano attestate altrove: donde il titolo del presente contributo, escogitato per segnalare la circostanza nonché per evidenziare la natura di scrigno della città che ospita il documento.

Questo studio focalizza la sua attenzione essenzialmente sui testi verbali, latini e francesi, delle sei composizioni uniche, adespote come tutte quelle ospitate nel manoscritto. Uno sguardo d'insieme sulla raccolta consente di fare il punto sulle ipotesi affacciatesi in merito alla sua origine prima e dopo l'unica, criticatissima trascrizione integrale, apparsa nel 1953 a corredo di una riproduzione in facsimile. I rapporti della fonte torinese con le due maggiori raccolte di mottetti duecenteschi (i manoscritti di Bamberg e di Montpellier) e con alcune fonti contenenti canzoni trovieriche con e senza musica contribuiscono a delineare lo scenario in cui la raccolta prese presumibilmente forma. Una ricognizione sulla pluralità di modelli presenti nella piccola collezione di conducti e nella più ampia collezione di mottetti consente di ipotizzare - rivedendo alcuni giudizi apparsi nel tempo nella letteratura musicologica - l'esistenza di una relazione semantica, e non solo di una giustapposizione spaziale, fra di esse.

Qualche linea di tendenza sembra emergere fin da questo sguardo preliminare: la presenza di testi sia latini sia francesi nei Tenores; la strutturazione musicale di questi sulla base o meno di modi ritmici; l'episodica adozione di questi ultimi anche nelle parti superiori; l'occasionale floridezza, convenzionalmente attribuita al magistero di Petrus de Cruce, della fisionomia del Triplum; il rarissimo reimpiego del testo e della musica del Tenor nel Motetus di un altro mottetto; la presenza non infrequente di episodi di scambio d'ambito fra voci (Stimmtausch); l'esistenza - in 
qualche caso rilevante - di armonie di terza, indicative di una prossimità dell'epoca di composizione dei pezzi rispetto a quella di compilazione del manoscritto. Limitate al gruppo ristretto degli unica, le considerazioni svolte in queste pagine potranno essere ampliate, raffinate ed eventualmente rettificate in sede di allestimento di un'edizione critica dell'intera raccolta, auspicabilmente da avviarsi in un prossimo futuro.

\section{Il manoscritto I-Tr Varia $42 / 2(=$ TO)}

Malgrado la sua importanza, affermata nel 1910 e sottolineata nel 1923 da Friedrich Ludwig, e malgrado l'edizione in facsimile e la trascrizione integrale pubblicate nel 1953 da Antoine Auda, il manoscritto I-Tr Varia 42/2 (d'ora in poi: TO) ha ricevuto nel tempo attenzioni episodiche ${ }^{1}$; per lo più di riflesso, all'interno di studi dedicati alle grandi raccolte mottettistiche dell'Ars Antiqua, il codice di Bamberga (D-BAs, Lit. 115; d'ora in poi: BA) e il codice di Montpellier (F-MO, H 196; d'ora in poi: $\mathrm{MO})^{2}$. Dei trentuno mottetti presenti in TO, infatti, dodici si trovano attestati in BA e addirittura venticinque (i dodici di $\mathrm{BA}$, più altri tredici) in $\mathrm{MO}$; donde la loro menzione nella letteratura critica prevalentemente in quanto concordanze di composizioni ospitate nelle due raccolte maggiori ${ }^{3}$

(1) F. Ludwig, Repertorium organorum recentioris et motetorum vetustissimi stili, 2 voll., a c. di L.A. Dittmer, New York, The Institute of Mediaeval Music, 1978, I/2, Handschriften in Mensuralnotation, cap. IX, pp. 533-546; Id., Die Quellen der Motetten ältesten Stils, “Archiv für Musikwissenschaft" 5, 3, luglio 1923, pp. 185-222 (una descrizione sintetica e un indice dei contenuti di TO si trovano alle pp. 205206) e 5, 4, gennaio 1924, pp. 273-315; A. Auda, Les 'motets wallons' du manuscrit de Turin, Vari [sic] 42, 2 voll., Bruxelles, chez l'auteur [1953].

(2) Di ambedue i codici esistono moderne edizioni critiche, apparse verso la fine degli anni Settanta e tuttora adoperate in sede di riflessione critica e di pratica esecutiva: Compositions of the Bamberg Manuscript, Bamberg, Staatsbibliothek, Lit. 115 (olim ED. IV. 6), a c. di G.A. Anderson, Stuttgart, American Institute of Musicology-Hänssler, 1977, «Corpus Mensurabilis Musicae», 75; The Montpellier Codex, 4 parti, a c. di H. Tischler, Madison (WI), A-R Editions, 1978. Le due edizioni riprendono, aggiornano e migliorano quelle prodotte nei primi decenni del Novecento, rispettivamente Cent motets du XIII siècle, publiés d'après le manuscrit Ed. IV. 6 de Bamberg, 2 voll., eds. P. Aubry, Paris, Rouart, Lerolle et Co.-Geuthner, 1908 e Polyphonies du XIII siècle: le manuscrit H 196 de la Faculté de Médecine de Montpellier, 4 voll. ed. Y. Rokseth, Paris, Dyer, 1936-39. Subito dopo la sua comparsa, l'edizione preparata negli anni Trenta e pubblicata dopo la guerra da Auda è andata invece incontro a critiche diffuse: si vedano le recensioni di M. Bukofzer, "Notes" 11, 2, marzo 1954, pp. 266-268; D. Fr. De Meeûs - A. Goosse, "Scriptorium" 8, 1, 1954, pp. 164-167; L.A. Dittmer, "Die Musikforschung" 10, 1, 1957, pp. 189-195. Malgrado le riserve espresse da diversi studiosi ormai più di sessant'anni fa, nessuno ha sinora messo in cantiere una nuova edizione di TO. Questo studio costituisce un primo tentativo in tale direzione.

(3) F. Gennrich, Trouverelieder und Motettenrepertoire, "Zeitschrift für Musikwissenschaft" 9, 1, ottobre 1926, pp. 8-39 e 9, 2, novembre 1926, pp. 65-85; H. Besseler, Studien zur Musik des Mittelalters. II. Die Motette von Franko von Köln bis Philipp von Vitry, "Archiv für Musikwissenschaft" 8, 2, gennaio 1927, pp. 137-258; G.A. Anderson, A Small Collection of Notre Dame Motets ca. 1215-1235, "Journal of the American Musicological Society" 22, 2, estate 1969, pp. 157-196; J. Stenzl, Eine unbekannte Sanctus-Motette vom Ende des 13. Jabrbunderts, "Acta Musicologica" 42, 3-4, luglio-dicembre 1970, pp. 128-138; W.E. Dalglish, The Use of Variation in Early Polyphony, "Musica Disciplina" 26, 1972, pp. 37-51; Th. Walker, Sui Tenor francesi nei mottetti del '200, "Schede medievali" 3, luglio-dicembre 1982, pp. 309-336; P.P. Norwood, Performance Manuscripts from the Thirteenth Century?, "College Music Symposium" 26, 1986, pp. 92-96; Ead., Evidence Concerning the Provenance of the Bamberg Codex, "The Journal of Musicology" 8, 4, autunno 1990, pp. 491-504; E. Schreurs, Music at the Collegiate Church of Tongeren and the School of Liège in the Late Middle Ages, "Revista de Musicología" 6, 4, 1993, pp. 2476-2494; K. Desmond, New Light on Jacobus, Author of "Speculum musicae", "Plainsong and Medieval Music" 9, 1, 2000, pp. 19-40; J.-Fr. Goudesenne, L'office de s. Winoc de Bergues (Flandres, XI' siècle) est-il à l'origine d'une teneur dans les motets du XIII siècle? Recherche sur la teneur 'Aptatur' dans les motets des manuscripts de Montpellier et de Bamberg, "Journal of the Alamire Foundation" 4, 2000, pp. 283-295; M. Everist, Motets, French Tenors, and the Polyphonic Chanson ca. 1300, "The Journal of Musicology" 24, 3, estate 2007, pp. 365-406; M. Bent, Magister Jacobus de Ispania, Author of the "Speculum musicae", Farnham (Surrey), Ashgate, 2015; R.C. Wegman, Jacobus de Ispania and Liège, "Journal of the Alamire Foundation" 8, 2, 2016, pp. 253-274. 
TO costituisce la porzione finale di un volume formato da due unità codicologiche, la prima delle quali (Varia 42/1: un manoscritto, a sua volta pergamenaceo, contenente un'antologia di testi dell'Antico Testamento preceduta da un'epistola di san Gerolamo) sicuramente collegata all'abbazia liegese di San Giacomo, prova ne sia l'annotazione «Liber sancti Jacobi in Leodio» vergata sul margine superiore del foglio iniziale ${ }^{4}$. I due manoscritti sono rilegati sin da fine Settecento in un unico volume recante sul dorso la dicitura «CODIC. M. S. | MEMBRANAC I EXIMIAE | RARITATIS | EX BIBLIOTHECA | CEL. ABATIE | ST. JACOBI | LEODIENSIS | I». Il numero romano è spia di una vicenda un po' complicata ma sintetizzata con efficacia in un inventario redatto intorno al 2000 da una funzionaria della Biblioteca Reale:

Gli attuali mss. Varia 42, 43, 44 e 45 facevano originariamente parte di un unico codice composito, smembrato e suddiviso in più volumi verso fine Settecento, con tutta probabilità ad opera di Carlo Ignazio Montagnini, conte di Mirabello e ambasciatore del re di Sardegna a L'Aja, che lo acquistò per sé nel 1788, quando fu posta in vendita la biblioteca dell'abbazia di Saint Jacques di Liegi. Probabilmente per un banale errore del rilegatore l'ordine dei volumi, impresso in numeri romani da I a IV sul dorso, sconvolse l'assetto originario del codice, che si recupera ordinando i volumi nel modo seguente: IV (Varia 45), II (Varia 43), III (Varia 44) e I (Varia 42) [...] Il Montagnini raccolse una scelta e varia libreria che venne posta in vendita nel 1819 dai cugini ed eredi Carlo e Girolamo Montagnini e fu fatta acquistare dal re Vittorio Emanuele I. È quindi verosimile che i quattro volumi siano rimasti dal 1790 al 1819 nella biblioteca di casa Montagnini a Trino (Vercelli), per diventare poi proprietà dei Savoia e pervenire infine alla Biblioteca Reales.

L'asta del marzo 1788 a cui fa cenno l'estratto dall'inventario è documentata dalla pubblicazione del relativo catalogo ${ }^{6}$ l'eventualità che Montagnini vi abbia preso parte, assicurandosi i manoscritti poi rilegati nei quattro volumi attualmente posseduti dalla Biblioteca Reale, è testimoniata da alcune note apposte di suo pugno sui fogli di guardia del terzo e del quarto ${ }^{7}$. Ambasciatore sabaudo nelle Province Unite

(4) Fondata nell'XI secolo e passata attraverso alterne vicende nei secoli successivi, l'abbazia fu secolarizzata e trasformata in collegiale sulla base di un breve di Pio VI datato 28 maggio 1785; una delle conseguenze di questo fatto fu lo smembramento della biblioteca, istituzione i cui volumi furono messi in vendita tre anni dopo.

(5) Inventario del Fondo Varia, a c. di A. Terracina [dattiloscritto inedito; Torino, Biblioteca Reale, ca. 2000], s.v. A Montagnini è dedicata un'ampia voce nel vol. 75 (2011) del Dizionario Biografico degli Italiani, Roma, Istituto dell'Enciclopedia Italiana, 1960-. In relazione agli interessi culturali dell'ambasciatore, da essa si evincono notizie interessanti in merito ai suoi rapporti con alcuni bibliofili e letterati del tempo come Tommaso de Ocheda e Carlo Denina. Una ricostruzione più remota e meno facilmente accessibile, ma solo di poco meno dettagliata delle vicende del codice originario, si trova in J. Miethke, Ein neuer Text zur Geschichte der politischen Theorie im 14. Jahrbundert: der "Tractatus de potestate Summi Pontificis" des Guillelmus de Sarzano aus Genua, "Quellen und Forschungen aus italienischen Archiven und Bibliotheken” 54, 1974, pp. 510-38: 511-514.

(6) Catalogue des livres de la bibliothèque de la célèbre abbaye de St-Jacques à Liège dont la vente se fera publiquement au plus offrant, sur les cloîtres de laditte ex-abbaye, le 3 mars 1788 et jours suivans [...], Liège, 1788. Il catalogo è commentato in S. Balau, La bibliothèque de l'abbaye de Saint-Jacques à Liège, "Bulletin de la Commissione Royale d'Histoire” LXXI, 1902, p. 42 e ss. Un altro catalogo (N. Bouxhon, Summa omnium quae in bibliotheca inferiori Sancti Jacobi continentur [1667], B-Br, 13993) documenta, più di un secolo prima, la presenza del codice nella medesima biblioteca. Più in generale le vicende dei manoscritti posseduti nel tempo dall'abbazia benedettina liegese sono sintetizzate in Chr. Mortiaux-Denoël, Le fonds des manuscrits de l'abbaye de Saint-Jacques de Liège, "Revue bénédictine" 101, 1991, pp. 154-191 e in Ead. - E. Guillaume, Le fonds des manuscrits de l'abbaye Saint-Jacques de Liège. II. Dispersion et localisation actuelle, "Revue bénédictine" 107, 1997, pp. 352-380.

(7) Il fatto che le note sui due fogli di guardia siano di pugno di Montagnini è confermato dal confronto con un'altra nota, sicuramente autografa, apposta sul manoscritto "Storia patria 735" della Biblioteca Reale. 
da un decennio dopo aver trascorso molti anni rivestendo incarichi di natura diplomatica fra Vienna e gli stati tedeschi, Montagnini aveva messo insieme una biblioteca ragguardevole; in questo quadro s'inserisce la sua partecipazione all'asta liegese ${ }^{8}$. Rientrato a Torino nel 1789, Montagnini morì all'improvviso nell'estate dell'anno seguente; essendo celibe, suoi eredi divennero i cugini avvocati che nel 1819 vendettero parte dei suoi beni documentari alla casa reale.

La ricostruzione dell'ordine in cui i manoscritti si succedevano prima dello smembramento del codice e della loro nuova rilegatura nei volumi poi ospitati dalla biblioteca di Montagnini è stata resa possibile dalla permanenza di una paginazione continua da 1 a 249 ${ }^{\circ}$. Nel volume I (= Varia 42) la numerazione copre tutti i fogli della prima unità codicologica e si arresta sul primo foglio della seconda, ovvero di TO. Il manoscritto musicale reca infatti due diverse paginazioni: una antica, in numeri romani scritti con inchiostro rosso, relativa alla sola sezione dei mottetti (I - XXXVI), e una moderna, in numeri arabi scritti a matita, inglobante anche i fogli precedenti, contenenti i conducti $([1]-41)^{10}$. La mancanza di continuità con la paginazione che allineava i manoscritti precedenti nel codice originario, nonché la sua unicità di manoscritto musicale in un codice formato da documenti di altra natura, insinua qualche dubbio in merito al rapporto di TO con la vita culturale dell'abbazia. I dubbi discendono da due ordini di considerazioni, l'assenza nelle pagine di TO di ogni riferimento non solo a San Giacomo, ma alla città di Liegi, e il genere non solo non sacro, ma in qualche caso addirittura parodistico, testimoniato nella raccolta di mottetti ${ }^{11}$.

Se la conservazione di TO nella biblioteca dell'abbazia liegese di San Giacomo sino al 1788 è un fatto assodato, oggetto di obiezioni è stata la tesi dell'origine vallona dei mottetti. Sostenuta da Auda sin dal titolo della sua edizione, a motivo delle venature dialettali riscontrabili nei testi verbali in lingua volgare, essa suscitò fin da subito le riserve di un filologo romanzo come André Goosse; il quale - nato e cresciuto nel cuore delle Ardenne a differenza del collega musicologo, francese del Dipartimento della Loira - la contestò senza nemmeno escludere che il manoscritto avesse avuto origine nell'Île de France ${ }^{12}$. Costrette nello spazio angusto di un'appendice a una recensione, le osservazioni di Goosse ebbero il merito di raccogliere l'appello

(8) Una stima approssimativa della numerosità della biblioteca di Montagnini va dai 3000 ai 4000 volumi. Cfr. G.A. De Gregory, Istoria della vercellese letteratura ed arti, 4 voll., Torino, Chirio e Mina, 1819-24, vol. IV, 1824, pp. 248-253 e la Relazione del Piemonte del segretario francese Sainte-Croix, con annotazioni di Antonio Manno, «Miscellanea di Storia Italiana» XVI, 2a serie, t. 1, Torino, 1877, nota 77.

(9) IV (= Varia 45), ff. 1-42; II (= Varia 43), ff. 43-107; III (= Varia 44: i numeri di pagina sono oggi mancanti a causa dell'asportazione dei margini dei fogli in occasione di un'antica rifilatura; ma il numero dei fogli - 61, presumibilmente numerati da 108 a 168 - è congruente con la ripresa della numerazione nel volume successivo); I (= Varia 42), ff. 169-249.

(10) Questa circostanza ha indotto qualche esegeta a ipotizzare che in origine la raccolta dei conducti fosse indipendente da quella dei mottetti, cfr. E.M. Maschke, "Deus in adiutorium intende" Revisited: Sources and Contexts, in The Montpellier Codex: The Final Fascicle. Contents, Contexts, Chronologies, a c. di C.A. Bradley e K. Desmond, Woodbridge (Suffolk)-Rochester (NY), Boydell and Brewer, 2018, pp. 100120: 116; per una diversa lettura del problema $v$. infra n. 21. In ogni caso, al fine di armonizzare le due paginazioni RISM B IV/1 (Manuscripts of Polyphonic Music, 11th-Early 14th-Century, a c. di G. Reaney, München-Duisburg, Henle, 1966, pp. 801-07) attribuisce le lettere da [A] a [E] alla sezione contenente $\mathrm{i}$ conducti, in modo tale da produrre l'equivalenza [1]-41 = ([A]-[E]) + (I - XXXVI). La ricerca di informazioni relative a TO nelle varie basi dati facenti leva su RISM, prima fra tutte DIAMM (https://www.diamm. ac.uk/sources/185/\#/; ultimo accesso 7 giugno 2019), è resa difficoltosa dalla perpetuazione da parte di Reaney dell'errore commesso da Auda nel riportare la sigla del manoscritto ("Vari" anziché "Varia").

(11) F. Ludwig, Repertorium cit., pp. 533-534. Il tratto parodistico del genere mottettistico è sintetizzato nel titolo di uno degli studi più ampi e perspicui sull'argomento: S. Huot, Allegorical Play in the Old French Motet. The Sacred and the Profane in Thirteenth-Century Polyphony, Stanford (CA), Stanford University Press, 1997.

(12) De Meeûs - Goosse, "Scriptorium” 8, 1, cit., pp. 166-167. 
lanciato indirettamente da Ludwig, il quale nel 1923 aveva rilevato come neppure i testi verbali degli unica di TO fossero stati ancora editi ${ }^{13}$. Nel 1954 l'occasione era stata appena persa da Auda; il quale, rinunciando a ogni indagine filologica, nella sua trascrizione si era limitato a riprodurre - non senza incorrere in una serqua di errori, puntualmente rilevati da Goosse - i testi verbali dei mottetti al disotto dei rispettivi pentagrammi. Proponendo un'edizione annotata dei testi del conductus e dei cinque mottetti attestati unicamente in TO, questo studio tenta di colmare la lacuna segnalata da Ludwig ormai quasi un secolo fa.

\section{La collezione dei conducti}

TO s'inaugura con tre conducti, uno esteso ("Parce, virgo", ff. [A]r-[D]r), e due più brevi costruiti sul medesimo testo ("Deus in adiutorium", f. [D]v e f. [E]r) ${ }^{14}$ :

Deus in adiutorium

intende laborantium

ad doloris remedium

festina in auxilium,

ut chorus noster psallere

possit et laudes dicere

tibi Christe rex gloriae

gloria tibi Domine ${ }^{15}$.

Quella di associare alle raccolte mottettistiche una versione polifonica di "Deus in adiutorium", un tropo rimato del versetto inaugurale del Salmo 70 (69) introduttivo delle ore dell'ufficio, era una prassi diffusa nel Medioevo. Le due brevi intonazioni di TO - una più antica, basata su cantus firmus (f. $[\mathrm{E}]^{\mathrm{r}}$ ), e una più recente, tradizionalmente priva di fondamento nel repertorio del cantus planus (f. [D] ${ }^{v}$ ) - registrano concordanze in $\mathrm{BA}$, in $\mathrm{MO}$ e in tre codici di rilevanza e notorietà minori ${ }^{16}$ : il conduc-

(13) F. Ludwig, Die Quellen cit., pp. 205-206.

(14) Oltre che nelle citate edizioni critiche delle fonti che li recano individualmente, i tre conducti di TO sono trascritti e pubblicati in Notre Dame and Related Conductus, 9 voll. a c. di G.A. Anderson, Henryville (PA), Insitute of Mediaeval Music, 1978-1988, IX (Three-part Conductus in Related Sources), pp. 19-24. Per ogni indagine sul genere del conductus è oggi disponibile anche la base dati Cantum pulcriorem invenire (http://catalogue.conductus.ac.uk; ultimo accesso 7 giugno 2019).

(15) Con una strofa ulteriore («In te, Xpiste, credentium I miserearis omnium, I qui es Deus in secula | seculorum, in gloria») intercalata fra le due di TO il testo del tropo si trova intonato monofonicamente anche in un ufficio per la Festa della Circoncisione scritto dall'arcivescovo di Sens Pierre de Corbeil (ca. 1150-1222) e conservato nel manoscritto F-SEm 46, f. 2; cfr. H. Villetard, Office de Pierre de Corbeil; improprement appelé "Office des fous" d'après d'un manuscrit de Sens, XIII siècle, Paris, Picard, 1907, p. 87. Trascritta a p. 131, la melodia su cui è intonata ognuna delle tre strofe corrisponde a quella della voce inferiore (de facto, il Tenor) del terzo conductus di TO (f. [E $\left.]^{r}\right)$. Sulle fonti delle intonazioni mono- e polifoniche di diverse versioni di "Deus in adiutorium", tropo la cui origine risale all'undicesimo secolo, cfr M.Ch. Caldwell, A Medieval Patchwork. Song: Poetry, Prayer and Music in a Thirteenth-Century Conductus, "Plainsong \& Medieval Music" 25, 2, ottobre 2016, pp. 139-165: 150-159.

(16) Tutti pergamenacei, i manoscritti meno cospicui e meno noti sono: 1) D-DS, 3471 (d'ora in poi: DA), un frammento contenente una ventina di mottetti quasi tutti su testo latino; 2) B-Br, 19606 (d'ora in poi: BXL), una collezione di nove mottetti tutti su testo latino. 3) F-CA, Inc. B 165 (d'ora in poi: CA), un frammento contenente cinque composizioni appartenenti a generi diversi. Uno studio recente di BXL, fonte databile al 1334-35 e collegabile all'abbazia benedettina di Malmédy, centro a una trentina di chilometri a sud-est di Liegi, si trova in K. Kügle, Two Abbots and A Rotulus: New Light on Brussels 19606, in 'Quomodo cantabimus canticum?' Studies in Honor of Edward H. Roesner, a c. di D.B. Cannata, G.I. Currie, R.C. Mueller e J.L. Nádas, Middleton (MI)-Stuttgart, American Institute of Musicology-Hänssler, 
tus più antico inaugura il primo fascicolo di MO (f. 1), DA (f. 1a) e il corpus non mottettistico di BA (f. $62^{\mathrm{v}}$ ); il conductus più recente inaugura l'ottavo fascicolo di $\mathrm{MO}^{17}$ (f. 350), BXL (ff. 1-2) e si trova sul f. II di CA.

Spoglie, concise e notate in ordine cronologico inverso, le due intonazioni di "Deus in adiutorium" sono precedute in TO da quella di un appello rivolto alla Vergine: adornato da estesi melismi, il conductus "Parce, virgo" (ff. $[\mathrm{A}]^{\mathrm{r}}-[\mathrm{D}]^{\mathrm{r}}$ ) inaugura il manoscritto nel segno della "spes reorum" impreziosendolo con la sua opulenza, la sua ampiezza e - fatto non trascurabile - la sua unicità. Eccone il testo:

Parce, virgo, spes reorum, penitenti servulo;

ipsum solvens a suorum

delictorum vinculo.

Potes enim, tantum velis: ergo parce, domina, ad quid namque te fidelis, nisi forent crimina,

Invocaret, nec tu fores tanto digna filio, $\mathrm{Ni}$ fuissent peccatores et patrum transgressio.

Etsi penam culpa poscit, culpam delet venia, cum sit maior, que ignoscit, quam is, cui fit gratia.

\section{$\mathrm{A}<\mathrm{men}>^{18}$}

La differenza rispetto al breve testo che innerva gli altri due conducti è palese. Laddove "Deus in adiutorium" si articola in due strofe, la prima resa densa dai suoi versi tutti in "-ium", la seconda inizialmente più ariosa ma suggellata da una ridondante ("rex gloriae I gloria...") espressione di gloria, "Parce, virgo" si distende sontuoso, alternando le sue rime su un arco di lunghezza doppia; ovvero diffondendo l'invocazione alla Vergine su quattro strofe che le caudae, in numero di cinque, incor-

2008, pp. 145-185. A un mottetto costituito da una voce tropata sovrapposta alle tre della versione più recente del conductus "Deus in adiutorium" (CA, f. II) è dedicato un articolo di U. Günther, Les versions polyphoniques du "Deus in adiutorium", "Cahiers de civilisation médiévale" 31, 1988, pp. 111-122.

(17) D'ora in poi i diversi fascicoli di MO saranno indicati mediante sigle, da MO1 a MO8.

(18) Il testo verbale è trascritto seguendo i criteri editoriali utilizzati in Analecta Hymnica Medii Aevi, 55 voll., Leipzig, Reisland, 1886-1922, vol. 20 (Cantiones et Muteti I Lieder und Motetten des Mittelalters, a c. di G.M. Dreves, 1895), in cui il testo è trascritto a p. 169. L'unica fonte ivi citata, con una segnatura non altrimenti nota ("Cod. Taurinen. Reg. 11, ol. St. Jacobi Leodien.") è TO. Il testo verbale dovette godere in seguito di una certa fortuna nell'Europa luterana: in una versione logicamente non mariana («Parce, Christe, spes reorum...») e con l'aggiunta di altre quattro strofe esso risulta intonato a due voci nelle Piae cantiones ecclesiasticae et scholasticae veterum episcoporum, a c. di J. Finno, Greifswald, 1582 (1625/2), un'antologia più volte ripubblicata le cui origini sono rintracciabili nella scuola di canto della cattedrale di Ảbo (oggi Turku), Finlandia. Un'edizione d'uso è stata recentemente posta online dal curatore, Mats Lillhannus (http://www.lillhannus.net/piae-cantiones/; ultimo accesso 7 giugno 2019). 
niciano esperendo soluzioni ogni volta diverse ${ }^{19}$. Le espansioni più cospicue sono la prima e l'ultima, ovvero quelle che aprono e chiudono la composizione; adottando la tecnica dello boquetus, l'ultima cauda compie sul ritmo di sesto modo un'operazione raffinata, esemplificativa di una tendenza stilistica rilevata a suo tempo dall'Anonimo IV e divenuta oggetto di uno studio recente ${ }^{20}$.

Degno di nota è inoltre un legame musicale con l'intonazione più recente di "Deus in adiutorium", conductus con cui "Parce, virgo" condivide l'attacco, ovvero il contenuto della prima perfezione. Sebbene limitato nel tempo, il rimando s'impone all'ascolto per via della sua posizione esordiale. Inaugurandosi nel segno di un conductus costruito sulla formula principe dell'ufficio, "Parce, virgo" stabilisce un rapporto forte fra la figura del Creatore e la figura della Vergine, erigendole a custodi della raccolta mottettistica; la quale, come il trittico dei conducti, s'inaugura con una composizione di conio mariano $^{21}$.

Con la sua ricchezza verbale e musicale e col rimando esplicito alla successiva intonazione di "Deus in adiutorium", "Parce, virgo" mette il lettore sull'avviso del fatto che quello che ha davanti è un libro importante. La sua natura di appello alla Vergine per un atto di clemenza nei confronti dei rei è strettamente collegata a una delle funzioni assolte dal tropo "Deus in adiutorium" nella prassi liturgica e devozionale del Medioevo: quella, accreditata da Agostino, di talismano contro le tentazioni:

[Diabolus t] entare ergo non cessat; insidiari non cessat. Infrenatus est atque illigatus in cordibus impiorum, ne sæviat in Ecclesiam, et tantum faciat quantum vult. Fremunt dentes impiorum adversus dignitatem Ecclesiæ et pacem Christianorum, et quia non habent quid agant sæviendo; saltando, blasphemando, luxuriando, non impellunt corpora Christianorum, sed lacerant animas Christianorum. Clamemus ergo una voce omnes verba haec: Deus, in adjutorium meum intende. Opus enim habemus sempiterno adjutorio in isto saeculo. Quando autem non modo tamen in tribulatione positi maxime dicamus: Deus, in adjutorium meum intende $e^{22}$.

\section{La collezione dei mottetti}

Gli indici dei libri motetorum sono strutturati di norma in modo alfabetico ${ }^{23}$. Questo non è il caso di TO, manoscritto che ospita una raccolta il cui indice, verosi-

(19) Collocato prima dell'intonazione della prima strofa, il primo melisma non è a rigor di termini una cauda, ma un *caput. Tutti diversi l'uno dall'altro, i cinque segmenti sine littera comprovano - come del resto i quattro cum littera - un distanziamento deliberato della forma musicale, orientata all'invenzione continua, da quella letteraria, rispettosa dell'omogeneità strofica. Un cenno al conductus cum caudis che inaugura TO si trova in M. Everist, Discovering Medieval Song: Latin Poetry and Music in the Conductus, Cambridge, Cambridge University Press, 2018, p. 247. Dello stesso autore si segnala, in merito al genere del conductus cum caudis, anche un contributo precedente: Reception and Recomposition in the Polyphonic "Conductus cum caudis": The Metz Fragment, "Journal of the Royal Music Association" 125, 2000, pp. 135-163.

(20) S. Guhl-Miller, The Imperfect Rhythmic Modes of Anonymous IV: Deciphering his Sixth Mode(s), "Theoria" 22, 5, 2015, pp. 5-40: 31-37.

(21) Basato sul Tenor "<Vir->Go", il primo mottetto esordisce nel Motetus con le parole "Eximium decus virginum reorumque consolatio"; dunque, individuando come il conductus "Parce, virgo" un appiglio per i rei nella figura della Vergine (definita "consolatio" nel mottetto e "spes" nel conductus). Questo legame intertestuale induce a formulare una tesi opposta a quella di Maschke (v. supra, n. 10) in merito all'originale assenza di rapporto in TO fra la raccolta di conducti e quella di mottetti. Una riflessione più ampia su questo problema sarà compiuta in sede di edizione critica.

(22) Agostino, Enarrationes in Psalmos, in Patrologiae cursus completus: series latina, 221 voll. a c. di J.P. Migne, [Paris, chez l'auteur], 1844-66, XXXVI, col. 867.

(23) Cfr. M. Everist, French Motets in the Thirteenth Century. Music, Poetry, and Genre, Cambridge, Cambridge University Press, 1994, p. 10. 
milmente redatto a notazione ultimata, si limita a dichiarare l'ordine in cui le trentuno composizioni si succedono. Sul verso del f. [E], quello sul cui recto si trova il terzo e ultimo conductus, TO elenca per ciascun mottetto l'incipit del Motetus, associandolo all'indicazione del relativo foglio scritta - analogamente a quanto avviene sul margine superiore del recto dei fogli seguenti - in numeri romani con inchiostro rosso. Al di là della presenza di un denominatore comune costituito dall'impianto a tre voci, la raccolta non appare riconducibile a un modello tipologico definito ${ }^{24}$ ma si presenta come una raffinata crestomazia, ovvero una silloge basata su rapporti verbali, musicali e concettuali più o meno palesi ${ }^{25}$ [cfr. Tabella 1].

Osservando la tabella, il dato che balza all'occhio è la costruzione su T latino dei primi quattordici mottetti della collezione; ad essi fanno seguito tre mottetti su T francese (nn. 15-17); quindi due serie (nn. 18-20 e 21-23) costituite ognuna da due mottetti su T latino e da uno su T francese; infine otto mottetti (nn. 24-31) costruiti alternatamente su $\mathrm{T}$ francese e su $\mathrm{T}$ latino. Laddove su $\mathrm{T}$ francese è costruito un unico tipo di mottetto, le cui voci presentano tutte testi in lingua francese $(\mathrm{F} / \mathrm{F} / \mathrm{F}$ : nn. $15,16,17,20,23,24,26,28,30)$, su T latino sono costruiti mottetti caratterizzati da tutti e quattro gli assetti linguistici, ovvero: un mottetto integralmente latino (L/L/L: n. 5); tre che associano un $\operatorname{Tr}$ francese a un M latino (F/L/L: nn. 1, 10, 25); tre che fanno l'opposto $(\mathrm{L} / \mathrm{F} / \mathrm{L}: \mathrm{nn} .3,14,27)^{26}$; quindici - quasi metà della collezione - che presentano un Tr e un M entrambi francesi (F/F/L: nn. 2, 4, 6, 7, 8, 9, 11, 12, 13, 18, $19,21,22,29,31)$.

In rapporto a $\mathrm{BA}$ e a $\mathrm{MO}$ il dato saliente di $\mathrm{TO}$ è tuttavia la percentuale elevata di mottetti su $\mathrm{T}$ francese. Laddove questo tipo di composizione è attestato in BA solo in 3 casi su 99 ( $=3,33$ per cento) e - limitando la ricognizione ai soli fascicoli contenenti mottetti a tre voci - in non più di 25 casi su 234 ( $=10,68$ per cento $)$ in $\mathrm{MO}$, in TO esso s'afferma in 9 casi su 31 (=29,03 per cento). A tutta prima sorprendente, il dato di TO dev'essere però confrontato con quello relativo al solo corpus novum di MO (27,17 per cento): su 50 mottetti, infatti, MO7 ne presenta 15 su T francese, ammontanti al 30 per cento del totale; e pochi di meno ne presenta MO8, fascicolo in cui i mottetti su $\mathrm{T}$ francese sono $10 \mathrm{su} 42(=23,8 \text { per cento })^{27}$.

(24) Volgendo per un attimo lo sguardo alle due raccolte mottettistiche più ampie, si osserva come al netto di tre eccezioni l'ingente corpus di BA sia costituito di sole composizioni a tre voci, e come a specifiche tipologie di mottetti siano dedicati cinque degli otto fascicoli di MO. Oltre al conductus antico rispecchiato in TO e a sette clausulae, BA contiene due mottetti a due voci, novantanove a tre e uno a quattro. MO dedica ai mottetti a tre voci tre dei sei fascicoli del suo corpus antiquum (MO1-MO6, repertorio risalente all'incirca agli anni 1200-1270), distinguendoli per assetto (le lingue dei testi delle singole voci - F = francese, $\mathrm{L}=$ latino - sono elencate nell'ordine Triplum / Motetus / Tenor; d'ora in poi rispettivamente $\mathrm{Tr}$ / M / T): MO3: L/F/L; MO4: L/L/L; MO5: F/F/L. I restanti fascicoli del corpus antiquum (MO1, MO2, MO6) presentano rispettivamente una raccolta di composizioni di vario tipo (conductus, mottetto-boquetus, boquetus, organum), una di mottetti a quattro voci (F/F/F/L) e una di mottetti a due voci su T francese (L/F). I due fascicoli del corpus novum (MO7 e MO8, repertorio risalente all'incirca agli anni 1270-1300), quelli in cui i mottetti di TO trovano il maggior numero di concordanze, presentano invece - al netto del conductus inaugurale di MO8, rispecchiato in quello più recente di TO - due raccolte di composizioni a tre voci dagli assetti linguistici diversi.

(25) Lillustrazione di un'ipotesi relativa ai criteri di strutturazione della raccolta trascenderebbe i limiti e gli scopi del presente contributo. Essa sarà fornita nell'introduzione all'edizione critica.

(26) Conservati essenzialmente in F-Pn, n.a.fr. 13521 (La Clayette) e in MO3, i mottetti con Tr e M in lingue diverse costituiscono una componente cospicua di TO (6 composizioni su 31, quasi il 20\%).

(27) In BA sono costruiti su T francese i mottetti nn. 52, 53, 54; in MO7 i mottetti nn. 256, 260, 269, 270, 271, 272, 277, 280, 290, 291, 292, 294, 295, 297 e 302; in MO8 i mottetti nn, 309, 312, 313, 318, 319, $321,323,325,333,337$. Salvo il n. 302, ascrivibile al tipo L/F/F, tutti i mottetti elencati in questa nota appartengono al tipo $\mathrm{F} / \mathrm{F} / \mathrm{F}$, come i nove di TO. Su T francese $\mathrm{MO}$ propone anche un mottetto a quattro voci, il n. 25 (F/F/F/F: ma il T è il contrafactum di una melodia gregoriana). Un quadro sinottico dei mottetti su $\mathrm{T}$ francese conservati nei diversi manoscritti si trova in appendice a Walker, Sui Tenor francesi cit. 
Tabella 1

\begin{tabular}{|c|c|c|c|c|c|}
\hline \multirow[t]{2}{*}[\mathrm{N}.]{} & \multicolumn{2}{|c|}{ PAGINAZIONE } & \multicolumn{3}{|c|}{ INCIPIT } \\
\hline & romana & $a r a b a$ & Triplum & Motetus & Tenor \\
\hline 1 & $\mathrm{I}^{\mathrm{s}-\mathrm{II}^{v}}$ & $6^{\mathrm{r}}-7^{\mathrm{v}}$ & Or voi je bien k'il me covient & Eximium decus virginum & $<$ Vir $>$ Go \\
\hline 2 & $\mathrm{II}^{v}-\mathrm{III}^{\mathrm{v}}$ & $7^{v}-8^{v}$ & Entre Adam et Hanikel & Chies bien seans & Aptatur \\
\hline 3 & $\mathrm{IV}^{\mathrm{t}}-\mathrm{V}^{\mathrm{x}}$ & $9^{r}-10^{r}$ & Salve virgo virginum & Est ilh dont ensi & Aptatur \\
\hline 4 & $\mathrm{~V}^{i}-\mathrm{VI}^{t}$ & $10^{\mathrm{t}}-11^{\mathrm{r}}$ & Bele Aelis par matin & Herou herou je trahi & Flos filius \\
\hline 5 & $\mathrm{VI}^{\mathrm{r}}$ & $11^{\mathrm{r}}-11^{\mathrm{v}}$ & Iam nubes dissolvitur & Iam novum sidus oritur & Solem \\
\hline 6 & $\mathrm{VI}^{\mathrm{v}}$ & $11^{v}-12^{v}$ & Dame de valur et de bonteit & Héi diex cant je remir & Amoris \\
\hline 7 & VII"-IX & $12^{v}-14^{r}$ & Aul douch tens ke chantent & Biaus dous amis m'aveis & Manere \\
\hline 8 & $\mathrm{IX}^{\mathrm{v}}-\mathrm{XI}^{\mathrm{r}}$ & $14^{v}-16^{r}$ & Bien me doi soi tote riens & Je n'ai ken ke nus & Kyrieleison \\
\hline 9 & XI-XIIIr & $16^{\mathrm{r}}-18^{\mathrm{r}}$ & J'ai mis tote ma penseie & Je n'en puis mais & Puerorum caterva \\
\hline 10 & $\mathrm{XIII}^{\mathrm{r}}-\mathrm{XIV}^{\mathrm{r}}$ & $18^{\mathrm{r}}-19^{\mathrm{r}}$ & Aucun vont sovent & Amor qui cor vulnerat & Kyrieleyson \\
\hline 11 & $\mathrm{XIV}^{\mathrm{s}-\mathrm{XV}}$ & $19^{\mathrm{r}}-20^{v}$ & Aucun ont troveit & Lonc tens me sui tenus & Annuntiantes \\
\hline 12 & $\mathrm{XVI}^{\mathrm{r}}-\mathrm{XVII}{ }^{\mathrm{r}}$ & $21^{\mathrm{r}}-22^{\mathrm{r}}$ & Biaus dous amis & Grant pechiet fist cis & Vale \\
\hline 13 & XVII'-XVIII" & $22^{\mathrm{r}}-23^{\mathrm{v}}$ & Plus joliement c'onkes & Quant li dous tens & Portare \\
\hline 14 & $\mathrm{XVIII}^{v}-\mathrm{XIX}^{\mathrm{r}}$ & $23^{v}-24^{r}$ & O virgo pia candens & Lis ne glais ne rosiers & $<$ Amat $>$ \\
\hline 15 & $\mathrm{XIX}^{\mathrm{r}-\mathrm{XX}}$ & $24^{\mathrm{r}}-25^{\mathrm{r}}$ & Fine amurs ki les siens & J'ai lonc tens amurs servie & Orendroit plus c'onkes \\
\hline 16 & $\mathrm{XX}^{\mathrm{t}-\mathrm{XXI}^{\mathrm{r}}}$ & $25^{\mathrm{r}}-26^{\mathrm{r}}$ & Entre Copin et Borgois & Je me quidoie & Belle Izabelos m'at mort \\
\hline 17 & $\mathrm{XXI}^{v}-\mathrm{XXII}$ & $26^{\mathrm{v}}-27^{\mathrm{r}}$ & Sens penseir folur ai servi & Quant la saisons desireie & Qui bien aime a tart oblie \\
\hline 18 & XXII'-XXIII ${ }^{r}$ & $27^{\mathrm{r}}-28^{\mathrm{r}}$ & En mai cant rosiers florist & J'ai troveit ki m'amerat & Fiat \\
\hline 19 & $\mathrm{XXIII}^{+}-\mathrm{XXIV}^{t}$ & $28^{\mathrm{r}}-29^{\mathrm{r}}$ & Ki d'amur wet & Ki longuement poroit joir & Nostrum \\
\hline 20 & 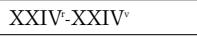 & $29^{\mathrm{r}}-29^{v}$ & Aul cuer ai unc mal & Je ne m'en repentirai & Jolietement moi tient \\
\hline 21 & $\mathrm{XXIV}^{\mathrm{v}}$-XXVII & $29^{v}-31^{v}$ & S'amours ewist point & Aul renouveleir dou jolit tens & Ecce iam votiva \\
\hline 22 & XXVIIr-XXVIII & $32^{r}-33^{r}$ & Je me doi bien doloseir & Por coi m'aveis vos doneit & Docebit \\
\hline 23 & $\mathrm{XXVIII}^{\mathrm{r}-\mathrm{XXX}}$ & $33^{r}-35^{r}$ & Qui amour wet & Li douls penseirs & Cis a cui je sui amiet \\
\hline 24 & $\mathrm{XXX}^{\mathrm{v}}-\mathrm{XXXII}^{\mathrm{r}}$ & $35^{v}-37^{r}$ & Diex ou porai je trouver & Ce sunt amorettes & Ki n'at point d'argent \\
\hline 25 & XXXII'-XXXIII" & $37^{\mathrm{r}}-38^{v}$ & Dous rossagnoles jolis & Virgo gloriosa & $\mathrm{L}<$ aetabitur $>$ \\
\hline 26 & 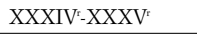 & $39^{r}-40^{r}$ & Leis l'ormelle en la turelle & Main soi levat sires garins & Je ne chaindrai mais \\
\hline 27 & $X_{X X V^{t}-X X X V I^{r}}$ & $40^{\mathrm{x}}-41^{\mathrm{r}}$ & Pulchra, decens, speciosa & Lonc tens at ke vi m'amie & Et sperabit \\
\hline 28 & $\mathrm{XXXVI}^{\prime}-\mathrm{XXXVII}{ }^{v}$ & $41^{\mathrm{r}}-42^{v}$ & Por ma dame de valur & La jolie penseie & $\mathrm{L}<$ is ne glais ne rosier $>$ \\
\hline 29 & XXXVII"-XXXVIII" & $42^{v}-43^{v}$ & Amours en cui j'ai mise & En mun cuer n'at pas tant & In seculum \\
\hline 30 & $\mathrm{XXIX}^{\mathrm{r}}-\mathrm{XL}^{\mathrm{r}}$ & $44^{r}-45^{r}$ & Tous solas et tote ioie & Bone amours ki les siens & Ne me blameis mie \\
\hline 31 & $\mathrm{XL}^{r}-\mathrm{XL}^{v}$ & $45^{\mathrm{r}}-45^{\mathrm{v}}$ & Hei diex, de si haut & Mal batus longuement & Cumque \\
\hline
\end{tabular}

La percentuale cospicua di mottetti su $\mathrm{T}$ francese è un dato collegabile alla posteriorità di redazione di $\mathrm{TO}$, manoscritto risalente al secondo decennio del Trecento, rispetto a BA (ca. 1260) e al corpus antiquum di MO (MO1-MO6: ca. 1260-80) ${ }^{28}$. Una conferma in questo senso viene dall'allineamento del dato percentuale di TO a quello di MO7 e MO8, fascicoli formanti quel corpus novum la cui redazione è databile al primo decennio del Trecento. Oltre ai tre soli mottetti su T francese (nn. 16, 20, 23)

(28) Oltre che mediante riscontri relativi al repertorio, la recente ipotesi di datazione di TO - di un paio di decenni più tarda rispetto a quella formulata dai primi esegeti (cfr. H. Besseler, Studien zur Musik, des Mittelalters. II., p. 142) - fa leva su indagini relative alla sua decorazione. Cfr. J. Olivier, Gotbic Manuscript Illumination in the Diocese of Liège (c. 1250 - c. 1330), 2 voll., Corpus van verluchte handschriften uit de nederlanden, Leuven, Peters, 1988; Ead., L’héritage de Philippe Bruni, Doyen du Chapître de la Sainte-Croix de Retour à Liège, "Bulletin de la Société d'art et d'histoire du Diocèse de Liège"LX, 1995, pp. 47-63; Everist, Motets cit., pp. 369-370. 
presenti in blocco in BA (nn. 52, 53, 54) $)^{29}$ e ripresi separatamente nel corpus novum di MO (nn. 256, 260, 280), TO ne annovera altri due (nn. 24, 30) assenti in BA ma presenti in MO (nn. 288, 318). Oltre a tre unica (nn. 15, 17, 28), TO accoglie infine l'unico mottetto su T francese (n. 26) assente sia in BA sia in MO ma presente in altri tre manoscritti, uno dei quali probabilmente, e due dei quali sicuramente posteriori ${ }^{30}$.

\section{I cinque unica}

Basati due su Tenor latino (nn. 12, 29) e tre su Tenor francese (nn. 15, 17, 28), i cinque unica di TO presentano voci superiori tutte dotate di testi francesi. Estratta dalla precedente, la tabella sottostante raccoglie i dati ad essi relativi:

Tabella 2

\begin{tabular}{|c|c|c|c|c|c|}
\hline$[\mathrm{N}]$. & \multicolumn{2}{|c|}{ PAGINAZIONE } & \multicolumn{3}{|c|}{ INCIPIT } \\
\hline & romana & araba & Triplum & Motetus & Tenor \\
\hline 12 & 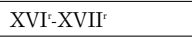 & $21^{\mathrm{r}}-22^{\mathrm{r}}$ & Biaus dous amis & Grant pechiet fist cis & Vale \\
\hline 15 & $\mathrm{XIX}^{\mathrm{r}}-\mathrm{XX}^{\mathrm{r}}$ & $24^{r}-25^{r}$ & Fine amurs ki les siens & J'ai lonc tens amurs servie & Orendroit plus c'onkes \\
\hline 17 & $\mathrm{XXI}^{\mathrm{v}}-\mathrm{XXII}^{\mathrm{r}}$ & $26^{\mathrm{v}}-27^{\mathrm{r}}$ & Sens penseir folur ai servi & Quant la saisons desireie & Qui bien aime a tart oblie \\
\hline 28 & $\mathrm{XXXVI}^{\mathrm{r}-\mathrm{XXXVII}}$ & $41^{\mathrm{r}}-42^{v}$ & Por ma dame de valur & La jolie penseie & $\mathrm{L}<$ is ne glais ne rosier $>$ \\
\hline 29 & $\mathrm{XXXVII}^{\mathrm{v}}-\mathrm{XXXVIII^{v }}$ & $42^{v}-43^{v}$ & Amours en cui j'ai mise & En mun cuer n'at pas tant & In seculum \\
\hline
\end{tabular}

Nelle pagine che seguono i testi verbali del Tr, del M e del T di ogni mottetto sono presentati sinotticamente, al fine di agevolare l'individuazione dei reciproci rapporti lessicali e semantici. Quanto alle peculiarità della loro intonazione, in questa sede sono forniti solo alcuni ragguagli destinati a futuri sviluppi in sede d'edizione critica.

In generale, l'analisi dei testi verbali permette di affermare che se l'ipotesi formulata da Auda nel 1953 circa l'origine piccarda della raccolta è senz'altro difficile da verificare, la scripta di TO per i cinque mottetti oggetto del presente studio mostra tratti linguistici tipici di quell'area, o comunque del nord dell'ambito d'oïl, sia a livello fonetico sia a livello morfologico; dunque si può supporre che la copiatura, quanto meno quella dei testi verbali, sia stata effettuata da un amanuense nativo di tale area; dove essa abbia avuto luogo, è un quesito che resta per ora privo di una risposta definitiva.

Per quanto riguarda il vocalismo, i fenomeni più significativi sono:

- l'evoluzione di a tonica in sillaba libera > ei (ex. poraleir, n. 15, M, v. 9; endureir, n. 12, Tr, v. 2; donreis, ibidem, v. 1; demoreie, n. 12, M, vv. 3, 7; etc. $)^{31}$;

(29) Eccezionalmente notati su tre colonne, i tre soli mottetti su $\mathrm{T}$ francese presenti in BA costituiscono un corpo estraneo all'interno del manoscritto, per il resto ospitante una raccolta di quasi cento mottetti tutti su T latino, le cui parti sono notate su una o due colonne.

(30) I tre manoscritti che attestano concordanze col mottetto n. 26 (F-CA, B 1328; I-IV, CXV; I-UDc, 290) non ne presentano alcuna con gli altri mottetti di TO: I-IV, CXV e I-UDc, 290 risalgono alla seconda metà del Trecento, mentre F-CA, B 1328 riunisce al suo interno fogli di origine e provenienza diversa; quello recante il mottetto in oggetto (f. $3^{\text {r }}$ ) è databile intorno al 1300.

(31) Ch.T. Gossen, Grammaire de l'ancien picard, Paris, Klincksieck, 1970, p. 47. Nel testo sono però presenti anche forme come entree, pree (n. 17, M, vv. 1,3) e coloree (n. 28, M, v. 9). 
- l'evoluzione di e breve tonica $+1>$-iau (biaus, n. 12, Tr, v. 1 e M, vv. 3, 9; n. 28, T, v. 4; biaul, n. 29, M, v. 7);

- la monottongazione in -i- di e breve tonica in sillaba libera ${ }^{32}$ (tinent, n. 29, Tr, v. 3);

- l'evoluzione di o chiusa tonica + nasale $>$ u (ex. mun, n. 15, M, v. 7; sun, ibidem, Tr, v. 7, bune, n. 15, Tr, v. 10 etc.) $)^{33}$;

- l'evoluzione di o lunga tonica in sillaba aperta > ou, u (ex. melhour, n. 17, Tr, v. 3; douchur, ivi, v. 8; onur, ibidem, v. 9, languour, n. 28, Tr, v. 16, etc.);

- l'evoluzione del dittongo au > o + yod in gaudia > joe (n. 15, Tr, v. 1) forse da accostare al fenomeno che riguarda o tonica + yod in parochia $>$ paroche, segnalato da Gossen ${ }^{34}$.

Per quanto concerne il consonantismo, i fenomeni più significativi sono:

- la mancata palatalizzazione del nesso velare sorda $\mathrm{k}+\mathrm{a}>\mathrm{k}$ (ex. cangier, $\mathrm{n} .12$, Tr, v. 3; kaul, n. 15 , T, v. $5^{35}$ );

- la palatalizzazione alla finale in corouch (n. 12, Tr, v. 3), di cui è registrato un esempio in $\mathrm{GDF}^{36}$ tratto da $Y$ de et Olive, racconto breve tràdito dal manoscritto I-Tn, L.II.14, di origine piccarda ${ }^{37}$ e, più tardi, nelle Chroniques di Froissart ${ }^{38}$; forse da accostare a formazioni verbali analogiche del tipo douch (douter), arch (ardoir), prench (prendre) ${ }^{39}$;

- la palatalizzazione di $\mathrm{t}+\operatorname{yod}>$ š in grasce (n. 12, Tr, v. 9), ancora attestato nelle Chroniques $^{40}$;

- la presenza di w iniziale, anche non di origine germanica (welh < volěo, n. 12, Tr, v. 2; n. 28, Tr, v. 3; wet< volět, ibidem, v. 8; welhe, ibidem, T, v. 6) ${ }^{41}$.

Quanto alla morfologia, si osservano in particolare:

- la forma del pronome personale tonico singolare P1 mi masch. (ex. n. 17, T, v. 6), P3 li masch. (ex. n. 12, M, v. 7) e P3 li femm. (ex. n. 17, T, vv. 9, 11) ${ }^{42}$;

(32) Ivi, p. 52.

(33) Ivi, p. 83.

(34) Ivi, p. 77.

(35) Ivi, p. 95; secondo Gossen, questo tratto separa piccardo e normanno dal resto dei dialetti della Francia settentrionale; nei testi qui in esame la forma palatalizzata è comunque ben rappresentata (chanteir, n. 15, Tr, v. 2 e ibidem, M, v. 1 et passim; chans, n. 28, T, v. 2; chançon, ibidem, v. 5).

(36) F. Godefroy, Dictionnaire de l'ancienne langue française et de tous ses dialectes $d u \quad I X^{e}$ au XV siècle, Paris, s. e., 1880-1902 (http://micmap.org/dicfro/search/dictionnaire-godefroy/; ultimo accesso: 7 giugno 2019)

(37) G. Giannini, Poser les fondements: lieu, date, contexte (Essai sur le recueil L.II.14 de Turin, "Études Françaises" 48, 3, 2012 (Lire en contexte: enquête sur les manuscrits de fabliaux), pp. 11-31 (https://doi. org/10.7202/1015388ar; ultimo accesso 7 giugno 2019).

(38) «[Li contes de Montfort] blasma durement monsigneur Hervi de Lyon, par courouch de chou qu'il les avoit si tost fait retraire» (J. Froissart, Chroniques, t. 2: 1340-1342, a c. di S. Luce, Paris, Vve J. Renouard, 1870 , p. 112, cit. in DMF 2015 s.v. courroux).

(39) Ch.T. Gossen, Grammaire cit., pp. 132, 133

(40) «Li rois d'Engleterre tint a amour les Flamens et ne lor osta nulles des grasces faites ne donnees en devant, mais les amplia tousjours en bien pour euls» (J. Froissart, Chroniques: Début du premier livre. Édition du manuscrit de Rome Reg. lat. 869, a c. di G.T. Diller, Genève, Droz, 1972, cit. in DMF 2015, s.v. grâce).

(41) Reine Mantou, Actes originaux rédigés en français dans la partie flamingante du comté de Flandre (1250-1350). Étude linguistique, Liège, Georges Michiels, 1972, pp. 227-228.

(42) Ch.T. Gossen, Grammaire cit., p. 124; Ph. Ménard, Manuel du français du Moyen Âge, 1. Syntaxe de l'ancien français, Bordeaux, Sobodi, 1976, p. 55. 
- il pronome dimostrativo soggetto sing. masch. cis (cis ki m'at marieie, n. 12, M, v. 1$)^{43}$;

- l'aggettivo possessivo P1, caso regime $\mathrm{men}^{44}$.

Dal punto di vista della forma, la letteratura critica sul mottetto ha sottolineato spesso l'irregolarità metrica e ritmica di questi tipi di composizione; il fenomeno si riscontra anche nei testi verbali oggetto di questo studio, per i quali - eccettuato un solo caso $^{45}$ - non è stato possibile ricostruire una forma metrica regolare. È possibile intravedere infatti la presenza di moduli ritmici che oscillano intorno a blocchi di dieci sillabe talvolta alternati a blocchi di sette, come nel Tr del n. 12 e del n. 29, oppure di abbozzi di versi di cinque sillabe, come nel M del n. 15 e del n. 28, o ancora moduli di circa otto sillabe, come nel $\mathrm{Tr}$ del n. 17. Anche per quanto riguarda la rima, tutti i testi presentano parole-rima con omeoteleuti che tuttavia non corrispondono a schemi consolidati dalla tradizione; per esempio, nel Tr del n. 17 sono presenti due rime $^{46}$, mentre nel Tr del n. 29 si alternano due rime intercalate da un solo verso con sillaba finale diversa ${ }^{47}$. Schemi a tre rime sono presenti invece nel $\mathrm{Tr}$ del $\mathrm{n} .12$ e nel T del n. $15^{48}$. Si sarebbe dunque tentati di accostare questi abbozzi di versi al tentativo di alcuni autori del Trecento francese di stabilire un legame fra verso e prosa secondo un progetto collegabile con la ricerca, sei secoli più tardi, di un poema in pros ${ }^{49}$; ma solo uno studio più approfondito sull'insieme dei testi di TO potrà verificare la fondatezza di questa ipotesi.

Nelle trascrizioni che seguono si è scelto di disporre i testi evidenziando la ricorrenza di suoni-rima, in modo tale da ricostruire un abbozzo di versificazione che tenga conto allo stesso tempo del ritmo e della struttura sintattica, senza forzare le frasi nell'alveo di una forma rigorosa. Le norme in uso per i testi in antico francese sono state seguite per la risoluzione delle abbreviazioni, per l'introduzione della distinzione $\mathrm{i} / \mathrm{j}, \mathrm{u} / \mathrm{v}$ e per la normalizzazione dell'uso delle maiuscole. L'accento acuto indica -e tonica in sillaba finale nelle parole polisillabe (ex. adés), la cediglia è stata introdotta per dare al grafema c il valore fonetico di [s] (ex. chançon). L'apostrofe indica una vocale elisa (ex. k'ilh). L'uso moderno della punteggiatura è stato introdotto per facilitare la lettura.

\subsection{Il mottetto n. 12: Biaus dous amis | Grant pechiet | Vale TRIPLUM}

1 Biaus dous amis, queil conseilh me donreis?

De mun marit le dangier ${ }^{50}$ ne welh ne ne pui plus endureir,

(43) Ch.T. Gossen, Grammaire cit., p. 128.

(44) Ivi, p. 125.

(45) Il M del n. 12 è costituito da dodici decasillabi con rima abaa bbab abab.

(46) N. 17, Tr: $i(e): \operatorname{our}(s)$.

(47) N. 29, Tr: ei(e): an (s) (con varianti grafiche), vermelhette v. 9, amurs v. 16. Più delicato è valutare la rima in casi come il $\mathrm{M}$ del n. 28 , a causa dell'impossibilità di stabilire con certezza se la -r finale sia muta o sonora.

(48) N. 12, Tr: eis: eir: ent; n. 15, T: ai $(t, s)$ : ans: is.

(49) Questa la tesi sostenuta da Sarah Kay per il Bestiaire d'Amours di Richard de Fournival (Chant et désenchantement dans le "Bestiaire d'Amours" de Richard de Fournival, in Plus agreable a lire en prose qu'en rime? Vers et prose en moyen français, numero monografico della rivista "Le Moyen Français" 76-77, 2015). Tutti gli articoli di questo fascicolo riflettono sul complesso rapporto tra verso e prosa in antico e medio francese.

(50) Dangier, s. m.: potere esercitato su qualcuno. 
car sovent le voi cangier ${ }^{51}$ de corouch, cant a vos me voit parleir.

4 Bien sai k'ilh est por vos enjalosi e entreis ${ }^{52}$, car ilh me soloit sovent rire et mult bellement ${ }^{53}$ a parleir, or le voi si crueilment ${ }^{54}$ vers moi soventes fois regardeir ke je croi ${ }^{55}$, mien esscient ${ }^{56}$,

8 s'ilh vos plaist, et vos wet agreeir ${ }^{57}$, ke nos en irons vriement entre mi et vos deporteir ${ }^{58}$, ou de mun marit, por Dieu, me delivreis.

\title{
MOTETUS
}

1 Grant pechiet fist cis ki m'at marieie cant ${ }^{59}$ plus ne puis demoreir avec vos, biaus dous amis, ke curte demoreie ${ }^{60}$

$4 \mathrm{ki}$ at si tost cornei la matineie ${ }^{61}$ : ne quidoie pas k'encores fuist jours. Or m'en irai avec cel, or ja l'o ${ }^{62}$, si li dirai ke ceste demoreie

8 ai fait anuit ${ }^{63}$ avec mes dous secours ${ }^{64}$. Biaus dous amis, a ceste bien aleie ${ }^{65}$ Mi baisereis une fois, et je vos. Ce poise moi $^{66} \mathrm{k}$ 'a vilain sui doneie 12 cant je ne puis oblieir mes amurs.

\section{TENOR}

\author{
Vale
}

(51) Cangier, v. intr.: alterarsi, mutare di sentimenti.

(52) Entreis, p. pass. in funzione agg.: forse l'equivalente di "belliqueux" (cfr. entrer DMF 2015 IA1a, attaquer un ennemi).

(53) Bellement, avv.: con dolcezza, con una buona disposizione d'animo.

(54) Crueilment, avv.: severamente.

(55) Croi: ind. pres. croire, P1 considerare probabile.

(56) Mien escient, loc.: in tutta coscienza.

(57) Agreeir a qn corrisponde a "convenire", "essere d'accordo"; qui dunque il senso è "se voleste essere d'accordo".

(58) Deporteir, v. tr.: divertirsi, amoreggiare.

(59) Cant, avv.: dato che.

(60) Demoreie, s. f.: lasso di tempo.

(61) Corner, v. tr.: annunciare, quindi "è già stato annunciato il mattino".

(62) $O$, ind. pres. P1 ö̈r, quindi "lo odo già" (DMF 2015, s.v. ouïr: «LUDIN. Il me semble que je les $o$ NACHON. Non sont, non» (Le Mystère de l'Incarnation et Nativité de notre Sauveur et Rédempteur JésusChrist, représenté à Rouen en 1474. Publié d'après un imprimé du XV siècle, a c. di P. Le Verdier, Rouen, impr. E. Cagniard, 1885, t. 2, p. 112.

(63) Anuit, avv.: questa notte. La frase significa letteralmente "ho fatto questa notte questo soggiorno", cioè "sono stata presso di voi questa notte".

(64) Il sostantivo secours è termine della retorica amorosa, ma qui sembra maggiormente riferirsi al significato che aveva nel diritto, in cui designava ciò che è utile alla difesa di qualcuno (FEW, Band XII, p. 383 s. v. succurrere); la dama indicherebbe quindi i suoi dolci argomenti di difesa del suo operato.

(65) Bien aleie è una formula di saluto che si usa prima del congedo, corrisponde ad "addio".

(66) Poiser, v. intr.: essere penoso per qn. 
Testo verbale e melodia del T provengono dal repertorio di cantus planus, nello specifico da "Ave regina caelorum", un'antifona facente parte dell'ufficio di alcune feste mariane. Eccone il testo:

Ave regina caelorum, ave domina angelorum, salve radix, salve porta ex qua mundo lux est orta, gaude virgo gloriosa super omnes speciosa, vale o valde decora et pro nobis Christum exora ${ }^{67}$.

Della melodia originaria il $\mathrm{T}$ adotta la porzione corrispondente all'intonazione delle parole "vale o valde decora l et pro", surrogando con una spoglia cadenza sulla finalis l'intonazione, analogamente ornata, di "nobis Christum exora". L'autore del mottetto innerva la melodia sul terzo modo ritmico, distendendola lungo 32 perfezioni e disponendone tre enunciazioni consecutive, per un totale di $(3 \times 32=) 96$ perfezioni.

$\mathrm{M}$ e Tr non mostrano affinità rilevanti col testo del T. L'antifona sembra essere stata adottata più per la sua natura musicale che per il suo contenuto semantico. Molto stretti sono invece i rapporti fra i testi verbali delle voci superiori, dominati dall'allocuzione "Biaus dous amis". Rivolta dalla donna all'amante, essa compare all'inizio del Tr e, per ben due volte, all'interno del M: nel Tr la donna si rivolge all'amante per chiedergli consiglio su come liberarsi dal marito («Biaus dous amis, queil conseilh me donreis? | de mun marit le dangier ne welh ne ne pui plus endureir»); nel M essa lamenta invece l'impossibilità di trattenersi con l'amante («plus ne puis demoreir avec vos, | biaus dous amis»), per poi dichiarargli l'intenzione di scambiarsi un bacio («Biaus dous amis, a ceste bien aleie $\mid \mathrm{m}$ 'i baisereis une fois, et je vos»).

Diffusa in molta lirica trovierica, la formula «Biaus dous amis» ricorre - creando uno dei tanti rapporti intertestuali interni alla raccolta - anche in un altro mottetto di $\mathrm{TO}$, il n. $7^{68}$. In quel caso l'allocuzione è pronunciata da una donna che, intendendo assicurarsi della fedeltà del suo amante reo di averla trascurata, instaura un dialogo con lui (M: «Biaus dous amis, m'aveis vos foit porteie I ki si longement m'aveis entroblieie? | Ma tres douce suer [...]»). A differenza di quanto avviene nel n. 12, nel n. 7 il rapporto intertestuale fra $\mathrm{M}$ francese e $\mathrm{T}$ latino esiste e si dimostra stretto. Il mottetto è costruito infatti su un $T$ che adotta un melisma su "manere", infinito che innerva il Verso del Graduale della Messa per la festa di san Giovanni Evangelista; virtualmente posta a fondamento di un mottetto, la frase «Sed sic eum volo manere, donec veniam, tu me sequere» costituisce l'bumus favorevole per lo sviluppo di un testo volgare incentrato sul tema della costanza.

Detto del fondamento su un T ripetuto, il mottetto risulta innervato sul terzo modo ritmico, ovvero su un modo la cui cellula, dispiegata su due perfezioni, lascia un margine ampio alle voci superiori per le loro rispettive elaborazioni. In conseguenza di ciò, le

(67) Corpus Antiphonalium Officii (d'ora in poi: CAO), 6 voll., a c. di R.-J. Hesbert, Roma, Herder, 1963-79, n. 1542. Una sintetica ricognizione sulle fonti, talvolta lievemente divergenti rispetto al testo edito in CAO, è offerta dalla base dati online Antiphonale Synopticum (http://gregorianik.uni-regensburg.de/ an/\#id/2167; ultimo accesso 7 giugno 2019).

(68) Il mottetto "Aul douch tens" "Biaus dous amis" | "Manere" (TO, n. 7) è presente anche in BA (n. 18) e nel corpus antiquum di MO (n. 119). 
colonne accordali sono ridotte della metà rispetto a quelle riscontrabili nei mottetti che adottano modi ritmici la cui durata corrisponde a una sola perfezione ${ }^{69}$. Associata alla configurazione del $\mathrm{T}$ in moduli di quattro perfezioni, l'ultima delle quali puntualmente coincidente con una pausa, la lunghezza pressoché analoga dei testi di Tr e M fa sì che il mottetto assuma la fisionomia musicale di un conductus. Un'ultima annotazione di carattere musicale riguarda il fenomeno dello Stimmtausch, ovvero dell'incrocio fra voci riscontrabile a volte nel repertorio di organa e conducti; assente in quello mottettistico

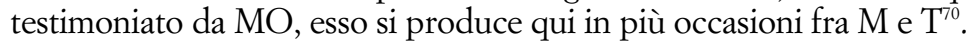

\subsection{Il mottetto n. 15: Fine amurs | J'ai lonc tens | Orendroit TRIPLUM}

1 Fine amurs ki les siens tient en joe ${ }^{71}$ me fait chanteir liement ${ }^{72}$, car elle m'at mis, soe mercit ${ }^{73}$, en voie $\mathrm{d}^{174}$ ameir bien

4 et loiaument pucelle simple et coie ${ }^{75}$, a cui valurs ${ }^{76}$ nulle autre ne se prent ${ }^{77}$ tant est sage et plaisans a tote gens, ke do sovenir de sun cors gent $\mathrm{t}^{78}$

8 ai si grant desduit jour et nuit ke riens ne m'anoie ${ }^{79}$ - donc mes cuers ${ }^{80}$ grasce rent sens faintise, doucement a bune amour qui sens sejour ${ }^{81}$ l'avoie a penseir si hatement $^{82}$.

\section{MOTETUS}

1 J'ai lonc tens amurs servie de chanteir, n'onkes ne me fut meri ${ }^{83}$, n'estant k'une foi ses dous oes ${ }^{84}$ turneir dengnaist ma damme ver mi

4 a cui dou tot me fait amur penseir.

(69) Lo stesso fenomeno si riscontra nel mottetto n. 14, "O virgo pia candens" I "Lis ne glais ne rosiers” “ “Amat”. Cfr. H. Besseler, Studien zur Musik des Mittelalters. II., p. 178.

(70) Limitando i rilievi alla prima enunciazione del T, il fenomeno si riscontra nelle perfezioni 4-6, 9-10 e 13-14.

(71) Tenir en joe: far rallegrare.

(72) Liement, avv.: con allegrezza.

(73) Soe mercit, in uso interiettivo con l'aggettivo possessivo, significa "grazie a lei" (DMF 2015 s.v. merci B3a).

(74) Mettre en voie de, loc: disporre, mettere nella disposizione d'animo di.

(75) Coie, agg.: discreta, riservata.

(76) Cui, caso regime indiretto del pronome relativo, ha qui il valore di complemento del nome e per questa ragione precede il sostantivo al quale si riferisce ("al valore della quale").

(77) Se prendre a qqn: assomigliare a qn.

(78) Gent, agg.: nobile.

(79) Anoier, v. tr.: affliggere.

(80) Mes è l'agg. poss. masch. caso soggetto sing. (C. Marchello-Nizia, La langue française aux XIV et $X V^{e}$ siècles, Paris, Nathan, 1997, p. 173) e il sostantivo corrispondente è declinato al nominativo singolare.

(81) Sans sejour: continuamente. Il relativo qui equivale a "io che" ed è da mettere in relazione con "riens ne m'anoie".

(82) Hatement, avv.: con sollecitudine.

(83) Merir, v. tr.: ricompensare.

(84) Oes, s.p.: occhi. Oel, forma attestata in GDF («Charlemaine de France fera les oes crever», tratto dalla Destruction de Rome). 
Las, por coi l'aim, cant onkes ${ }^{85}$ en li ne pous troveir riens ki moi fesist espereir mercit?

Et non porta $\mathrm{t}^{86}$, n'en puis mun cuer osteir

8 por mal endureir,

si sai bien a poraleir ${ }^{87}$

ke je morrai por li.

\section{TENOR}

1 Orendroit $^{88}$ plus c'onkes mais ${ }^{89}$ sens les mals d'amur plaisans, dous et amerus ${ }^{90}$ et gais,

4 por çoi je sui si joans ${ }^{91}$, si kaul' ${ }^{92}$ endurer ${ }^{93}$ me fait, et rire et chanteir et estre amis a belle dame a devis ${ }^{94}$

8 ki par sun douch regardeir fait mun cuer enamorer d'amour de pris ${ }^{95}$.

Questo mottetto è il primo dei tre unica costruito su T francese. Limitato in TO alla stanza iniziale, il testo verbale risulta attestato in cinque stanze nel manoscritto Douce 308 della Bodleian Library di Oxford, un documento non musicale redatto nell'area di Metz in una data posteriore al $1309^{96}$. Lo stesso testo si ritrova, sottoposto nella stanza inaugurale a una melodia in notazione mensurale, nel manoscritto Fonds français 1591 della Bibliothèque Nationale di Parigi ${ }^{97}$; benché non identici, melodia e testo verbale risultano simili a quelli del $\mathrm{T}$ del mottetto qui in esame. Affacciatasi presto nella letteratura critica, l'ipotesi che il mottetto citi la chanson non è stata esclusa da quella più recente; anzi, è stata rafforzata dalla constatazione che l'incipit del $\mathrm{M}$, «J'ai lonc tens amurs servie» evoca quello di un'altra chanson trovierica, Se j’ai lonc tens amours servi, attribuita a Gautier d'Epinal ${ }^{98}$.

(85) Onkes, avv.: mai.

(86) Non pourtant, avv.: tuttavia.

(87) A poraleir, loc. avv.: infine.

(88) Orendroit, avv.: adesso.

(89) Onkes mais, avv.: mai, in nessun momento.

(90) Amerus, agg.: innamorato. La forma è attestata nell'Anglo-Norman Dictionary (http://www.anglonorman.net/D/amerus; ultimo accesso 7 giugno 2019). In DMF 2015 s.v. amoureux la forma amereus è attestata nella Geste de Liège di Jean d'Outremeuse («Baptisiés fut Paris, li prinche sawereus; I Si fut nomeis Gondulphe, si en fut awireus, I Car li dus de Lotringe li fut si amereus, I Qu'il en fist son enfant plaisant et gratieus»).

(91) Joans, agg. s.: felice.

(92) Kaul, agg.: ardente in amore.

(93) Endurer, v. intr.: continuare a vivere (DMF 2015, s.v. endurer, II).

(94) A devis, loc.: a piacere, a volontà.

(95) De pris, loc.: di grande valore, prezioso.

(96) GB-Ob, Ms. Douce 308, ff. $171^{\mathrm{r}}-171^{\mathrm{v}}$. Nella letteratura critica il testo della chanson è contrassegnato dall'indicazione "Grand chant 67"; alcuni elementi della sua prima stanza compaiono anche nel Ballette 129, cfr. M. Atchison, The Chansonnier of Oxford Bodleian MS Douce 308: Essays and Complete Edition of Texts. Aldershot (Hants) - Burlington (VT), Ashgate, 2005 e Y. Plumley, The Art of Grafted Song: Citation and Allusion in the Age of Machaut, Oxford, Oxford University Press, 2013, pp. 49-52, 443.

(97) F-Pn, f. fr. 1591 (XIV sec.), ff. 64bisv-65v. La melodia è trascritta in G. Raynauds Bibliographie des altfranzösischen Liedes, 2 voll., Leiden, Brill, 1891, ed. mod. a c. di H. Spanke, Leiden, Brill, 1980, n. 197.

(98) Y. Plumley, The Art of Grafted Song cit., p. 51. Il solo testo verbale della chanson attribuita a Gautier 
A differenza di quanto avviene nel mottetto n. 12, qui il rapporto fra i testi verbali delle voci superiori e quello del T è stretto: $i$ tre testi costituiscono infatti altrettante variazioni sul medesimo tema, quello dell'impossibilità di sottrarsi al fascino femminile, e dunque all'impulso di elogiare la donna. I rapporti intertestuali sono intessuti non tanto su sintagmi ricorrenti, come nel caso precedente, ma sull'occorrenza di alcuni sostantivi-chiave come "amour" e "cuer" (entrambi, con grafie diverse, in $\mathrm{Tr}$, $\mathrm{M}, \mathrm{T})$ e di alcuni verbi come "chanteir" ( $\mathrm{Tr}, \mathrm{M}, \mathrm{T})$ ed "endureir" (M, T).

Il mottetto è il primo dei due unica su T francese di TO pubblicato prima dell'allestimento dell'edizione completa del manoscritto da parte di Auda; a metà degli anni Venti esso attirò l'attenzione di Gennrich nel quadro del suo studio dedicato ai rapporti fra chanson trovierica e repertorio mottettistico ${ }^{99}$. Alla stessa stagione risale anche un'osservazione di Besseler relativa ai rapporti armonici fra le linee vocali: la diffusa presenza di triadi è un elemento che induce a ritenere prossima all'epoca di compilazione del manoscritto l'origine della composizione ${ }^{100}$.

Anche se posta a fondamento di un mottetto, la melodia della chanson attestata in F-Pn, f. fr. 1591 mantiene il suo elevato grado di fluidità, ragion per cui il suo ritmo non è riconducibile a un modo specifico, quantunque appaiano con una certa frequenza istanze del secondo. La fluidità del $\mathrm{T}$ fa sì che la differenza di moto rispetto alle voci superiori sia minima, e che la composizione scorra via con un grado d'animazione costante. Sotto il profilo strutturale il $\mathrm{T}$ presenta all'inizio, in coincidenza coi quattro primi versi rimanti secondo lo schema abab, una doppia alternanza fra due frasi musicali identiche; dopodiché si sviluppa più liberamente, anche grazie al venir meno della regolarità del testo verbale. Il testo del $\mathrm{M}$ risulta intonato con riguardo alla struttura metrica e allo schema delle rime; non così quello del $\mathrm{Tr}$, che fluisce in modo più ornato, con un profilo melodico sostanzialmente svincolato da quello retorico.

\subsection{Il mottetto n. 17: Sens penseir | Quant la saisons | Qui bien aime TRIPLUM}

1 Sens penseir ${ }^{101}$ folur ${ }^{102}$, ai servi tote ma vie dou mond la melhour,

4 ne ja $\mathrm{a}^{103}$ por riens, com en die, ne ferai retur.

Cant je remir ${ }^{104}$ sa colur, lor moi semble k'elle rie

8 par douchur

d'Epinal è attestato nel cosidetto Chansonnier français de Saint-Germain des Prés (F-Pn, f. fr. 20050, ca. 1240-50), ff. $103^{\mathrm{r}}-103^{\mathrm{v}}$. Oggi visionabile online, il manoscritto fu pubblicato in facsimile e trascritto a fine Ottocento in M.P. Hyacinte - G. Raynaud, Le Chansonnier français de Saint-Germain des Prés, Bibl. nat. fr. 20050: reproduction phototypique avec transcription, Paris, Didot, 1892.

(99) F. Gennrich, Trouvèrelieder cit., pp. 67-69. L'altro unicum di TO ivi pubblicato è il n. 17 (pp. 69-72, v. infra).

(100) H. Besseler, Studien zur Musik des Mittelalters. II., p. 165. Le triadi di maggior durata si producono sulle seguenti perfezioni: 1 ( $\mathrm{T}=$ "Orendroit"), 3 ( $\mathrm{T}=$ "c'onkes"), 7 ( $\mathrm{T}=$ "plaisans"), 15 ( $\mathrm{T}=$ "joians"). Il fenomeno si riscontra ancor più diffusamente nel mottetto n. 17, v. infra.

(101) Penseir, v. tr.: preoccuparsi di qc., far caso a qc.

(102) Foulour, s. f.: sofferenza.

(103) Ne...ja: mai.

(104) Remirer, v. tr.: contemplare. 
et si moi permet l'onur ${ }^{105}$

k'amours

m'a fait desireir mains jour,

s'en oblie ma dolur.

\section{MOTETUS}

1 Quant la saisons desireie est entree ${ }^{106}$

k'ivers n'at pooir

et je voi por la vert pree ${ }^{107}$

4 la rosee sor la flur paroir,

lors sens main ${ }^{108}$ et soir

unc $^{109}$ mal ki m'agreie

c'om apelle desireir,

8 si plaisant a endureir

k'ilh moi fait ${ }^{110}$ chanteir.

\section{TENOR}

1 Qui bien aime a $\operatorname{tart}^{111}$ oblie, por ce ne puis oblier la plus belle et la melhor

4 ke j'onkes ${ }^{112}$ vis, si com est avis, ai $\mathrm{mi}^{113}$.

Tot mi penseir ${ }^{114}$ su $n$ t a li $^{115}$ et seront tos dis ${ }^{116}$,

8 tant com serai vis ${ }^{117}$.

Par ce, jointes mains li pri

ke je socur ${ }^{118}$ n'atende

d'autrui ke de li mercit ${ }^{119}$.

(105) Permettre ha qui il valore di "rendere possibile, realizzabile", mentre il sostantivo onour è l'equivalente di "favore, grazia accordata a qualcuno"; la frase significa quindi "se lei mi accorda il favore che amore mi ha fatto desiderare per tanti giorni" (per si ipotetico in afr., cfr. Ph. Ménard, Manuel cit., p. 235). Il $s$ ' che segue ha valore di sic latino ("dunque").

(106) Entrer, v. intr.: cominciare.

(107) Pree, s. f.: prateria.

(108) Main, s. m.: mattino.

(109) Unc, art. ind. m. s.: un.

(110) Font ] fait sulla base di tutti gli altri testimoni (F-Pn, f. fr. 1589, ff. 146"-147' ; F-Pn, f. fr. 1591, ff.

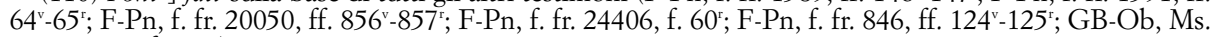
Douce 308, f. $\left.161^{v}\right)$.

(111) $A$ tart, loc. avv: raramente.

(112) Cfr. n. 83.

(113) Ai mi: ahimè.

(114) Sostantivo maschile a terminazione consonantica declinato al caso soggetto plurale.

(115) $L i$ in questo caso è pron. pers. femm. sing. caso regime (Th.C. Gossen, Grammaire cit., p. 124).

(116) Tos dis, loc. avv.: sempre.

(117) Vis, agg.: in vita.

(118) Socur, s. m.: aiuto, assistenza.

(119) De li mercit: li è pr. pers. ogg. P3 femm., mentre mercit, s. f., significa "misericordia", "grazia"; quindi, l'espressione equivale a "la sua misericordia". 
Oggetto dei tre i testi è il mal d'amore. Nel caso del $\mathrm{T}$ il conforto non può venire se non dalla donna stessa («je socur n'atende d'autrui | ke de li»); in quello del M il male è fonte di desiderio e dunque d'ispirazione per il canto (esso è «si plaisant a endureir | k'ilh moi font chanteir»); in quello del Tr il lenimento deriva dalla possibilità di continuare a rimirare e a servire la donna con la propria arte. Come nel caso del mottetto $\mathrm{n}$. 15, la composizione su $\mathrm{T}$ francese si segnala per la coesione semantica, ovvero per il rapporto stretto fra i suoi testi. Il principale elemento comune è il giudizio sulla qualità della donna, ritenuta la migliore del mondo (Tr: «dou mond la melhour») o per lo meno la più bella e la migliore mai vista dal poeta ( $\mathrm{T}$ : «la plus belle et la melhor ke j'onkes vis»), secondo un noto topos della lirica francese. Nel M la donna non è citata in modo esplicito, ma la sua presenza è surrogata da quella della rugiada che si deposita su un fiore, indicando l'avvento della stagione della reverdie, dunque della nascita dell'amore. Il tema del desiderio è il centro di gravità del mottetto: nelle sue varie concrezioni, dirette e indirette, esso compare nel Tenor ( $\mathrm{T}$ : «Tot mi penseir sunt a li»), nel Motetus (oltre che nell'incipit «Quant la saisons desireie», là dove quello d'amore è definito «une mal ki m'agreie | c'om apelle desireir»), e nel Tr («si moi permet l'onur k'amours | m'a fait desireir mains jour»).

$\mathrm{Al}$ netto di qualche variante per lo più ortografica il testo del $\mathrm{T}$ è quello di un proverbio molto diffuso nei manoscritti, musicali e non, del Medioevo ${ }^{120}$. Limitando la ricognizione ai manoscritti musicali che accolgono il proverbio in tutto o in parte, l'incipit «Qui bien aime tard oublie» è attestato in MO6 nel M di un mottetto a due voci ${ }^{121}$; inoltre, esso è intonato integralmente in diverse chansons à refrain, sempre però in modo diverso rispetto al mottetto in esame qui ${ }^{122}$. Il Tenor non influenza in TO la struttura della composizione; su di essa agisce in modo molto più evidente la melodia del $\mathrm{M}^{123}$. «Quant la saisons desireie» è una chanson anonima presente in molte fonti. Una è Méliacin (Conte du cheval de fust), un roman di Girard d'Amiens databile al 1285-1288: in F-Pn, f. fr. 1589 il testo compare in questi termini

\author{
Quant la sesons desirree est entree \\ qu'ivers na pooir \\ et je voi par la vert pree \\ la rosee lez la flor paroir, \\ lors sent main et soir
}

(120) Un'indagine recente sulla fortuna di questo proverbio nella cultura letteraria e musicale del Medioevo si trova in J. Saltzstein, The Refrain and the Rise of the Vernacular in Medieval French Music and Poetry, Cambridge, Brewer, 2013, pp. 71-72.

(121) MO, f. 240", n. 196, Biaus douz amis I Domine. Il verso "Qui bien aime a tart oublie" si trova all'interno del testo del $\mathrm{M}$.

(122) F-Pn, f. fr. 12483 ([Rosarius], ff. $1^{\mathrm{r}}-266^{\mathrm{v}}$; ca. 1330), f. 65 ; F-Pn, n.a. fr. 1050, f. 262 ; F-Pn, f. fr. 847, f. $194^{v}$; I-Rvat, Reg. lat. 1490 , f. $122^{v}\left(139^{v}\right)$. In quest'ultimo manoscritto, redatto in Artois tra fine Due- e inizio Trecento, il testo compare in una sezione (ff. 120-127) contenente quindici chansons mariane, quattordici delle quali attestate con musica. In accordo con la diversa destinazione, esso risulta modificato nei termini seguenti: «Kj bien aime a tart oublie I mais ne le puis oublier I la douce vierge Marie I de li me couvient chanter $\mid$ et me dis renouveler $\mid$ et faire de li m'amie | k'ele mi voelle acorder | a lui ki ne faut mie | tiens ki le voelent amer».

(123) H. Besseler, Studien zur Musik des Mittelalters. II. cit., p. 175. La melodia corrisponde a quella trascritta in G. Raynauds Bibliographie e contrassegnata dal n. 505. A proposito del M cfr. anche Huot, Allegorical Play cit., p. 219; J. Haines, Eight Centuries of Trobadours and Trouveres: The Changing Identity of Medieval Music, Cambridge, Cambridge University Press, 2004, p. 32; Manuscripts and Medieval Song: Inscription, Performance, Context, a c. di H. Deeming, E.E. Leech, Cambridge, Cambridge University Press, 2015, pp. 243-244. Una situazione contraria, ovvero un'influenza di un T dotato di testo verbale completo sulla struttura generale della composizione, si riscontra in TO nel mottetto n. 30 , discusso relativamente alla sua attestazione in MO in Everist, Motets, French Tenors cit., p. 398. 
un mal qui m'agree c'on apele desirrer, si plesant a endurer qu'i me fait chanter.

Esso è scritto con una spaziatura interlineare ampia, destinata a ospitare una notazione musicale su pentagrammi successivamente non tracciati ${ }^{124}$.

Al difuori del roman di Giraut d'Amiens, il testo compare - talvolta intonato anche in altre fonti: il già citato Douce 308 della Bodleian Library di Oxford e altri tre manoscritti custoditi nella Bibliothèque Nationale di Parigi ${ }^{125}$. Come già nel caso del M del n. 12, il manoscritto oxoniense presenta il solo testo verbale con una grafia per alcuni aspetti simile a quella di TO:

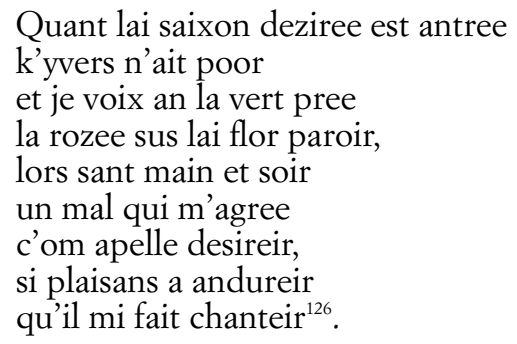

Tutti duecenteschi e dunque anteriori rispetto a Douce 308, i manoscritti conservati a Parigi presentano il testo in modo diverso. Il più antico dei tre (ca. 1240-50) è il già citato Chansonnier français de Saint-Germain des Prés, fonte che procura il testo sul verso di un foglio predisposto come altri per una notazione musicale successivamente non introdotta:

Quant la saisons desiree est entree

k'ivers n'ait pooir

et je voi au la verpree

la rousee sour la flor paroir,

lors sant men et soir

un mal ki m'agree

c'om apele desireir,

si plaisans a andurer

k'i me fait chan $<$ teir $>^{127}$.

(124) F-Pn, f. fr. 1589, ff. 146 $-147^{\mathrm{r}}$. Il roman è disponibile in un'edizione critica a c. di A. Saly, Girart d'Amiens. "Méliacin ou le Cheval de Fust", Senefiance 27, Aix-en-Provence, Publications du CUERMA, 1990. Secondo la lezione di un manoscritto conservato nella Biblioteca Riccardiana e Moreniana di Firenze (I-Fr, 2757) il testo del proverbio è trascritto anche da E. Stengel, Die altfranzösische Liedercitate aus Girardin d'Amiens "Conte de cheval de fust", "Zeitschrift für romanische Philologie" 10, 1886, pp. 460-476: 470, citazione n. 21. Il testo di Méliacin è presente anche in altri tre manoscritti, F-Pn, f. fr. 1455, F-Pn, f. fr. 1633 e B-Br, IV 319.

(125) Tutti appartenenti al Fonds français, i tre manoscritti recano rispettivamente i numeri di segnatura 20050, 24406 e 846 (l'ordine di elencazione corrisponde a quello cronologico di redazione, ovvero a quello in cui essi sono commentati in questo paragrafo).

(126) GB-Ob, Ms. Douce 308, f. 161" , col. dx.

(127) F-Pn, f. fr. 20050 (Le Chansonnier français de Saint-Germain des Prés), f. 124v. 
Redatto verosimilmente dopo il 1266, il secondo (F-Pn, f. fr. 24406) lo procura nella lezione seguente,

Quant la seson desirree est entree

qu'iver n'a pooir

et je voi par la vespree

la rousee seur la flour paroir,

lors sent main et soir

.i. mal qui m'agree

c'om apele desirrer,

si plesant a endurer

qu'i me fet chanter.

intonato su una melodia sensibilmente diversa rispetto a quella attestata in $\mathrm{TO}^{128}$. Redatto a Digione e databile all'ultimo quarto del Duecento, il terzo (F-Pn, f. fr. 846, detto Chansonnier Cangé) lo riporta in una lezione diversa,

Quant la saisons desirree est entree

qu'yvers n'a pooir

et je voi par la vert pree

la rosee sor l'erbe paroir,

lors sent main et soir

. i. mal qui m'agree

qu'en apele desirrer,

si plaisant a endurer

qu'i me fait chanter.

e soprattutto con una melodia praticamente identica a quella di $\mathrm{TO}^{129}$. La differenza più rilevante nel testo verbale è la variante relativa all'erba ("l'erbe"), che sostituisce nel quarto verso il fiore ("la flour", "la flor", "lai flor" nelle diverse grafie) presente in TO e in tutti i manoscritti qui in esame. Anche l'intonazione musicale, articolata in un neuma di più note seguito da una nota isolata, sembra addirsi maggiormente alla prosodia del sintagma "l'erbe" che a quella del sintagma "la flor"; nel valutare questo aspetto occorre tuttavia tener presente la differenza di stato e di funzione della melodia, indipendente nel caso della chanson e dipendente da un contesto polifonico in quello del mottetto.

In relazione al testo verbale del $\mathrm{Tr}$, l'unica osservazione riguarda l'incipit "Sens penseir folur", corrispondente a una formula ritrovabile in epoca posteriore nel v. 125 del Dit dou Vergier di Machaut ${ }^{130}$; limitatamente al sintagma “penseir folur", un'oc-

(128) F-Pn, f. fr. 24406, f. 60. La variante "vespree" in luogo di "vert pree" è significativa, poiché sostituisce un'indicazione di luogo (la prateria verde) con una di tempo (l'ora del vespro, la sera); essa è però verosimilmente erronea, considerando che la rugiada è fenomeno squisitamente notturno. Al termine della notazione della prima stanza si trova il testo della seconda, intonato limitatamente alla prima parola ("Douce", sulle stesse due note su cui comincia l'intonazione della prima) ma con l'intenzione di suggerire l'intonazione completa sulla stessa melodia a cui è sottoposto il testo della prima stanza.

(129) F-Pn, f. fr. 846, ff. $124^{\mathrm{v}}-125^{\mathrm{r}}$

(130) Le edizioni moderne di riferimento per l'opera letteraria di Machaut sono 1) Guillaume de Machaut, "The Fountain of Love" («La Fonteinne Amoureuse») and Two Other Love Vision Poems, a c. di e trad. R. Barton Palmer, New York - London, Garland (Garland Library of Medieval Literature, Series A: Texts and Translations, 54), 1993 (Le Dit dou vergier: pp. 22-86); 2) Euvres de Guillaume de Machaut a c. di E. Hoepffner, Paris, Champion pour la Société des anciens textes français, 1908-1921 (Le Dit dou 
correnza si riscontra anche all'interno di TO, nella parte finale del Tr del mottetto $\mathrm{n}$. 4 («plus me baterat ma mere | plus moi ferat penseir folur»).

Il mottetto n. 17 è il secondo dei due unica di TO pubblicati nel 1927 da Gennrich $^{131}$. L'immanenza nel mottetto della forma della chanson adoperata in funzione di $M$ è evidente in special modo nella prima parte, là dove una serie di tre idee melodiche corrispondente all'intonazione dei primi due versi è ripetuta integralmente sui successivi due. L'intonazione si dispiega in seguito in modo più libero, facendo assumere una forma meno regolare al mottetto. Il ritmo sostanzialmente riconducibile al secondo modo adottato dalla chanson si riflette nell'intonazione del proverbio nel T; il ritmo di secondo modo è invece assai meno avvertibile nel $\mathrm{Tr}$, parte melismatica che tende a disporre note distinte sul secondo e sul terzo tactus della perfezione. La presenza di triadi, già segnalata come tratto armonicamente innovativo nel mottetto $\mathrm{n}$. 15 , risulta qui ancor più diffusa; particolarmente fitte sono le occorrenze nella prima parte della composizione, quella la cui fisionomia è informata dalla struttura iterativa della melodia del M. Frequente e protratto per diverse perfezioni è sin dall'inizio il fenomeno dello Stimmtausch, favorito in primo luogo dall'ambito eccezionalmente ampio (una dodicesima, $\mathrm{do}^{3}-\mathrm{sol}^{4}$ ) in cui l'intonazione di "Qui bien aime a tart oublie" si dispiega nel $\mathrm{T}^{132}$.

\subsection{Il mottetto n. 28: Por ma dame | La jolie penseie $\mid L<i$ s ne glais $>$ \\ TRIPLUM}

1 Por ma dame de valur a cui sunt tuit mi penseir nuit et jour welh chanteir

4 et joie demeneir ${ }^{133}$, car tant est plainne de douchur, de cortoisie et d'onur, ke bien sai ke troveir

8 plus belle ne melhor ne porait om, sens doteir. Tant est plaisans a remireir, graciouse en parleir,

12 k'a briement conteir ${ }^{134}$, el mont ${ }^{135}$ n'at $\operatorname{sum}^{136}$ peir. Dieus, tant est nouble d'atur ${ }^{137}$ ke adés ${ }^{138}$ sui por s'amur

16 en torment et en languour, mais j'espoir de recovreir mercit des dous maus d'ameir

vergier: t. 1, pp. 13-56). Si veda inoltre D. Kelly, Machaut and the Medieval Apprenticeship Tradition: Truth, Fiction and Poetic Craft, Cambridge, Brewer, 2014, p. 29.

(131) F. Gennrich, Trouvèrelieder cit., pp. 69-72.

(132) Negli altri manoscritti in cui il proverbio compare intonato gli ambiti variano da una sesta $\left(\mathrm{do}^{3}-\mathrm{la}^{3}\right.$,

I-Rvat, Reg. lat. 1490, f. 122 $)$ a una decima $\left(\mathrm{fa}^{3}-\mathrm{la}^{4}, \mathrm{~F}-\mathrm{Pn}\right.$, n.a.fr. 1050 , f. $\left.262^{\mathrm{v}}\right)$.

(133) Joie demener, loc.: manifestare la gioia, rallegrarsi.

(134) Briement, avv:: in breve; il verso significa quindi "a dirlo in poche parole".

(135) Mont, s. m.: mondo.

(136) Sum, agg. poss. P3 caso regime.

(137) Atur, s. m.: aspetto.

(138) Adés, avv.: sempre, continuamente. 
k'elle me fait endureir;

20 prochainnement ma dolur

iert $^{139}$ aligie $^{140}$ et convertie sens demour ${ }^{141}$

en joie et en grant baudur ${ }^{142}$.

\section{MOTETUS}

1. La jolie penseie

ki me vient d'amur

me fait chanteir

4 por la plaisant $\mathrm{t}^{143}$ a vis cleir ${ }^{144}$

qui tant est seneie $^{145}$,

sage et aviseie ${ }^{146}$,

por ameir et joie demeneir ${ }^{147}$;

8 n'alhors ne puis penseir ${ }^{148}$, car sa face coloree ${ }^{149}$

ki tant fait a loeir

me fait a pou derveir ${ }^{150}$.

12 Sor totes autres ameie et servie et honoreie

doit estre, a droit parleir ${ }^{151}$.

Bien sai, sens douteir,

16 k'el mont n'at sun peir.

Bien doi amours honoreir cant j'ai, sens remueir ${ }^{152}$, a celle ke n'ous ${ }^{153}$ nomeir

20 mis do tot ${ }^{154}$ sunn penseir ${ }^{155}$, car s'amur tant m'agreie ke ja de lui ameir ne quier

men cuer desevreir ${ }^{156}$.

(139) Iert è la forma del futuro, P3 del verbo estre.

(140) Aligie, agg. f.: alleviata.

(141) Sans demour, loc. avv.: senza attendere, subito.

(142) Baudur, s. f.: allegrezza.

(143) Plaisant, agg. 'épicène', gentile.

(144) Vis cleir: bel viso.

(145) Seneie, agg. f.: assennata.

(146) Aviseie, p. p. in funz. agg.: prudente, saggia.

(147) Cfr. n. 133.

(148) Alhors penseir, loc. verb.: pensare ad altro.

(149) Coloree, agg. f.: dal bell'incarnato.

(150) Derveir, v. intr.: diventare matto.

(151) A droit parleir, loc. v.: a dire il vero.

(152) Sans remueir, loc. avv.: senza mutare parere.

(153) Ous, ind. pres. P1 di oser: oso.

(154) Do tot, loc. avv: pienamente, completamente.

(155) "La pensée d'elle", il pensiero di lei.

(156) Il verbo desevreir significa "separare"; il verso equivale a "non chiedo mai di distaccare il mio cuore dal fatto di amare" e quindi "di smettere di amarla". 


\section{TENOR}

$1 \mathrm{~L}<$ is ne glais ne rosiers floris ne chans d'oiselons, ne jolis mais $^{157}$, ne douce saisons,

4 biaus chans ne biaus dis, n'armonious sons mi font resjoïr ne faire chançon: tot ce mi font amours, welhe ou non,

8 ki m'ont mis en lor prison.>

Il T di questo mottetto corrisponde al $\mathrm{M}$ di un altro mottetto di TO: il n. 14, l'ultimo della lunga serie iniziale su T latino, "O virgo pia candens" | "Lis ne glais ne rosiers" | "<Amat $>$ " 158 . Eccezionale nei mottetti duecenteschi, lo scambio di funzione fra M e $\mathrm{T}$ ha la conseguenza di stabilire un rapporto fra le due composizioni: nel mottetto $\mathrm{n}$. 14 il testo amoroso in francese entra in rapporto con un $\mathrm{T}$ derivante dal Verso di un Responsorio per il secondo Vespro della festa di S. Caterina di Alessandria, "Sponsus amat sponsam salvator visitat illam”, e con un Tr latino di conio mariano:

$\begin{array}{lll}\text { TRIPLUM } & \text { MOTETUS } & \text { TENOR } \\ \text { O virgo pia } & \text { L<is ne glais ne rosiers floris } & \text { <Amat> } \\ \text { candens lilium } & \text { ne chans d'oiselons, ne jolis } \\ \text { super lilia } & \text { mais ne douce saisons, biaus chans } \\ \text { apud filium } & \text { ne biaus dis, n'armonious sons } \\ \text { regem omnium } & \text { m[o]i font resjoir ne faire chançon: } \\ \text { sis propicia } & \text { tot ce m[o]i font amours, welhe ou non, } \\ \text { ut deleas vicia } & \text { ki m'ont mis en lor prison> } \\ \text { peccatorum omnium } & & \\ \begin{array}{l}\text { et sanctorum gloria } \\ \text { nos collocet gratia }\end{array} & \end{array}$

Nel mottetto n. 28 esso diviene invece il fondamento di una composizione le cui voci superiori intonano entrambe testi profani in lingua francese. Come sempre tema centrale, l'amore vi è declinato in maniera diversa: nel $T$ esso è l'unica fonte d'ispirazione per un poeta sulla cui immaginazione nulla possono gigli, fiori, rose, canti di uccelli e belle stagioni; anche nel $\mathrm{M}$ esso è l'unica fonte d'ispirazione poetica, quantunque il testo non escluda con l'inflessibilità di quello del T l'esistenza di altre fonti; nel Tr l'amore arreca invece un dolore che può diventare a sua volta fonte di piacere.

Alla lunghezza ragguardevole dei testi di $\operatorname{Tr}$ e $M$, più che doppia rispetto a quella del T, corrisponde un'intonazione che in alcuni casi presenta gruppi di tre sillabe sottoposte ad altrettante semibrevi collocate su un unico tactus. Una tale frammentazione è prerogativa di pochi altri mottetti: i nn. 9 e 29 (v. infra) di TO e alcuni di quelli ospitati in MO7, il fascicolo del corpus novum con cui TO condivide quasi metà

(157) Jolis mais: il bel mese di maggio; la -s finale è indice della declinazione al caso soggetto singolare.

(158) $\mathrm{Il}$ mottetto è attestato in MO, ff. $227^{\mathrm{v}}-228^{\mathrm{r}}$, n. 177. Inoltre esso è presente anche in F-CA, Ms 410 A, f. $130^{v}$, manoscritto che lo riporta con alcune varianti, prima fra tutte la sostituzione del testo di "Lis ne glais ne rosier" con un testo mariano in latino ("O virgo Dei genitrix"). Rispetto al T del n. 14, il M del n. 28 presenta alcune varianti interessanti sotto l'aspetto musicale destinate a indagine in sede di edizione critica. 
del suo repertorio ${ }^{159}$. Come nei due casi precedenti, l'impiego nel $\mathrm{T}$ di una melodia tratta da una chanson trovierica comporta incroci ripetuti con le voci superiori, in un paio di casi addirittura col $\operatorname{Tr}$ (perfezioni 38 e 42). Numerose, in un mottetto di conio nuovo come questo, sono inoltre le triadi; una ventina, frequentemente aggiogate ma in due soli casi coincidenti col primo tactus di una perfezione (11 e 42, quella in cui si verifica il secondo caso di Stimmtausch fra T e Tr).

\subsection{Il mottetto n. 29: Amours en cui j'ai mise | En mun cuer | In seculum TRIPLUM}

1 Amours en cui j'ai mise ma penseie lonc tens, et ma douce damme la valhans me tinent jolit et envoisiet ${ }^{160}$ et gai,

4 por ce si en chanterai, car d'amerus ${ }^{161}$ cuer et vrai

la belle servirai trestot mun vivant.

Dieus, tant est mignotte et plaisans,

8 ke de li ameir n'iere ${ }^{162}$ ja repentans.

Cant je remir ${ }^{163}$ la bochette vermelhette, ses vairs ${ }^{164}$ ieus rians

si tres doucement,

12 sui aul cuer navrés, ke grant desirs ai de s'amur avoir, pour cui je sui en esmai,

16 mais se Dieus plaist et amurs, ke j'ai servie tos jours, je l'avrai.

\section{MOTETUS}

1 En mun cuer n'at pas tant de hardement ${ }^{165}$ ke j'ouse mostreir le mal ke je sens a la belle

4 a cui me sui doneis entierement.

Dieus, cant je primes ${ }^{166}$ regardai sun cors gent ${ }^{167}$, sun cleir vis ${ }^{168}$

et sun douch ris, sun biaul contennement ${ }^{169}$,

(159) I gruppi di tre sillabe sottoposti ad altrettante semibrevi su un unico tactus si trovano in sei perfezioni del $\operatorname{Tr}(3,20,22,30,36,44)$ e in sei del M $(8,18,20,24$, due nella 44). Cfr. anche H. Besseler, Studien zur Musik, des Mittelalters. II. cit., p. 165.

(160) Envoisiet, agg. m. s.: allegro.

(161) Cfr. n. 88.

(162) Iere, ind. fut. P1 di estre, 'sarò'

(163) Cfr. n. 102.

(164) Vairs, agg. m. pl.: blu-grigi, vivaci e chiari.

(165) Hardement, s. m.: audacia, coraggio.

(166) Primes, agg. in uso avv.: per la prima volta.

(167) Cfr. n. 76.

(168) Cfr. n. 142

(169) Contennement, s. m.: aspetto. 
8 herou $^{170}$, si tres doucement sui d'amours sopris ${ }^{171}$ c'onkes puis ${ }^{172}$

n'ous $^{173}$ talent $^{174}$ de penseir alhours ${ }^{175}$

$12 \mathrm{k}$ 'a li servir tous jours loiaument.

\section{TENOR}

\section{In seculum}

L'ultimo unicum di TO adotta uno dei T latini più ricorrenti nel repertorio duecentesco, soprattutto nei mottetti le cui voci superiori recano testi di argomento amoroso: ricavata dal Verso del Graduale della Messa di Pasqua («Confitemini Domino, quoniam bonus: quoniam in saeculum misericordia eius»), la locuzione avverbiale è inseribile agevolmente in testi profani incentrati su temi come la fedeltà e la determinazione nel servire la donna ${ }^{176}$. Al termine del Graduale a cui il Verso è collegato («Haec dies, quam fecit Dominus: exsultemus et laetemur in ea») spicca la presenza di una voce del verbo "laetor", ossia del verbo che innerva i Versi a cui appartengono i melismi su cui sono basati i due mottetti su T latino che precedono il presente ${ }^{177}$.

Le voci superiori stabiliscono all'interno di TO un legame con le voci corrispondenti nel mottetto n. $9^{178}$ : nel caso del M l'incipit dell'uno ( $\ll$ En mun cuer n'at pas tant de hardement») somiglia al secondo verso dell'altro («en mun cuer n'at se tristece»); nel caso del Tr la somiglianza si ravvisa invece fra gli incipit, rispettivamente «Amours, en cui j'ai mise ma penseie lonc tens» e «Jai mis tote ma penseie lonc tens en amur». Più della somiglianza, peraltro legata all'occorrenza di formule diffuse, nel caso delle voci superiori del mottetto n. 29 interessa un'evidente differenza, quella nella lunghezza. Doppia rispetto a quella del M, la lunghezza del Tr induce a ravvisare nel mottetto tratti petroniani; nondimeno, nell'intonazione del $\mathrm{Tr}$ la fluidità ritmica tipica del mottetto petroniano risulta assente, non essendovi traccia di raggruppamenti irregolari di note veloci. Il fenomeno che viceversa si segnala sin dalla prima perfezione è la sottoposizione, già riscontrata nei mottetti nn. 9 e 28 , di tre sillabe ad altrettante semibrevi insistenti sul medesimo tactus ${ }^{179}$.

Anche la fisionomia del $\mathrm{T}$, ripetitiva e stabilmente ancorata al secondo modo ritmico, risulta estranea al mottetto petroniano ${ }^{180}$. Connotato in senso innovativo è invece l'aspetto armonico, essendo 15 delle 50 perfezioni basate su aggregati com-

(170) Herou è un'interiezione che esprime angoscia, richiesta di aiuto.

(171) Etre sopris de l'amour de qqn significa "essere sedotti da qualcuno".

(172) Ne... onkes puis, loc. avv.: mai più da quel momento.

(173) Ous, ind pass. rem. P1 di avoir: ebbi.

(174) Avoir talent de, loc. v.: augurarsi

(175) Cfr. n. 145.

(176) Un'indicazione relativa alla diffusione del T "In seculum" nel repertorio di mottetti e conducti deriva dal fatto che esso ricorre in 8 delle composizioni contenute in BA e in ben 33 (con concentrazione massima nel quinto e nel sesto fascicolo e con latitanza totale nel settimo) di quelle contenute in MO.

(177) Il mottetto n. 25 è basato sul T "L<aetabitur>", melisma ricavato dall'intonazione del Verso dell'Alleluia della Messa della Quinta domenica dopo la Pentecoste, "Domine in virtute tua laetabitur rex et super salutare tuum exsultabit vehementer"; il mottetto n. 27 è basato invece sul T "Et sperabit", melisma ricavato dall'intonazione del Verso del Commune unius martyris "Laetabitur iustus in Domino et sperabit in eo et laudabuntur omnes recti corde".

(178) Il mottetto n. 9 di TO è attestato anche in MO6 (n. 238).

(179) Oltre che nella prima, il fenomeno si riscontra nel Tr in due perfezioni contigue $(14,15)$.

(180) Intessuto su 22 perfezioni, il T presenta una ripetizione integrale (perfezioni 23-44) e una ripetizione parziale (perfezioni 45-50). 
prendenti un intervallo di terza ${ }^{181}$. Limitato a una sola occorrenza fra M e T (perfezioni 3-4), il fenomeno dello Stimmtausch si produce in diverse occasioni, e talora per lunghi tratti (perfezioni 20-23, 39-41), fra Tr e M.

\section{All'orizzonte}

Alzando per un attimo lo sguardo dal novero degli unica, questo studio può fornire in epilogo qualche elemento utile per una messa in relazione di TO con le fonti che trasmettono qualcuno dei suoi mottetti. La Tabella 3 raccoglie i dati relativi alle concordanze con le raccolte di Bamberg e di Montpellier:

Tabella $3^{182}$

\begin{tabular}{|c|c|c|c|c|c|}
\hline \multicolumn{2}{|c|}{ TO } & \multicolumn{2}{|c|}{$B A$} & \multicolumn{2}{|c|}{$M O$} \\
\hline n. & ff. & n. & ff. & n. & ff. \\
\hline 1 & $6^{\mathrm{r}}-7^{v}$ & 32 & $16^{v}-17^{v}$ & 273 & $303^{r}-304^{v}$ \\
\hline 2 & $7^{v}-8^{v}$ & 24 & $13^{\mathrm{r}}-14^{\mathrm{r}}$ & 258 & $280^{v}-282^{r}$ \\
\hline 3 & $9^{r}-10^{r}$ & 33 & $18^{\mathrm{r}}-18^{\mathrm{v}}$ & 268 & $295^{v}-297^{r}$ \\
\hline 4 & $10^{\mathrm{r}}-11^{\mathrm{r}}$ & 42 & $23^{r}-23^{v}$ & 94 & $132^{v}-134^{r}$ \\
\hline 5 & $11^{\mathrm{r}}-11^{\mathrm{v}}$ & & & 275 & $306^{v}$ \\
\hline 6 & $11^{v}-12^{v}$ & 39 & $22^{\mathrm{r}}$ & 281 & $318^{r}-318^{v}$ \\
\hline 7 & $12^{v}-14^{r}$ & 18 & $9^{v}-10^{v}$ & 119 & $163^{v}-166^{r}$ \\
\hline 8 & $14^{v}-16^{r}$ & & & 262 & $286^{\mathrm{r}}-288^{\mathrm{r}}$ \\
\hline 9 & $16^{\mathrm{r}}-18^{\mathrm{r}}$ & & & 255 & $275^{v}-277^{v}$ \\
\hline 10 & $18^{\mathrm{r}}-19^{\mathrm{r}}$ & & & 264 & $290^{v}-292^{r}$ \\
\hline 11 & $19^{\mathrm{r}}-20^{\mathrm{v}}$ & & & 254 & $273^{r}-275^{r}$ \\
\hline 13 & $22^{\mathrm{r}}-23^{\mathrm{v}}$ & & & 257 & $279^{r}-280^{v}$ \\
\hline 14 & $23^{v}-24^{r}$ & & & 177 & $227^{v}-228^{r}$ \\
\hline 16 & $25^{\mathrm{r}}-26^{\mathrm{r}}$ & 52 & $31^{v}-32^{r}$ & 256 & $277^{v}-279^{r}$ \\
\hline 18 & $27^{\mathrm{r}}-28^{\mathrm{r}}$ & & & 320 & $368^{v}-370^{v}$ \\
\hline 19 & $28^{r}-29^{r}$ & & & 19 & $23^{v}-25^{r}$ \\
\hline 20 & $29^{\mathrm{r}}-29^{\mathrm{v}}$ & 53 & $32^{r}-32^{v}$ & 260 & $283^{v}-284^{v}$ \\
\hline 21 & $29^{v}-31^{v}$ & & & 253 & $270^{r}-273^{r}$ \\
\hline 22 & $32^{\mathrm{r}}-33^{\mathrm{r}}$ & & & 243 & $265^{v}-266^{r}$ \\
\hline 23 & $33^{\mathrm{r}}-35^{\mathrm{r}}$ & 54 & $32^{v}-34^{r}$ & 280 & $314^{\mathrm{r}}-317^{\mathrm{v}}$ \\
\hline 24 & $35^{v}-37^{r}$ & 22 & $12^{\mathrm{r}}-12^{\mathrm{v}}$ & 288 & $324^{v}-326^{r}$ \\
\hline 25 & $37^{\mathrm{r}}-38^{\mathrm{v}}$ & & & 38 & $68^{v}-71^{r}$ \\
\hline 27 & $40^{\mathrm{r}}-41^{\mathrm{r}}$ & 57 & $35^{v}-36^{r}$ & 78 & $116^{v}-118^{r}$ \\
\hline 30 & $44^{\mathrm{r}}-45^{\mathrm{r}}$ & & & 318 & $367^{v}-368^{v}$ \\
\hline 31 & $45^{\mathrm{r}}-45^{\mathrm{v}}$ & 63 & $39^{v}-40^{v}$ & 92 & $130^{v}-132^{r}$ \\
\hline
\end{tabular}

(181) Il mottetto franconiano presentava al massimo un paio di intervalli di terza, mentre quello petroniano ne contava di norma da 5 a 10; il numero superiore di occorrenze riscontrabili nel mottetto n. 29 induce a ritenerlo frutto di una fase successiva. H. Besseler, Studien zur Musik des Mittelalters. II. cit., p. 161.

(182) Dalla tabella sono esclusi i righi relativi ai mottetti nn. 12, 15, 17, 28, 29, unica e dunque privi di concordanze, e quello relativo al mottetto n. 26 , le cui concordanze si trovano - caso unico in tutta la raccolta - solo al difuori di BA e di MO. 
Come detto in apertura, dei trentuno mottetti di TO dodici trovano concordanze in BA e venticinque (i dodici di BA, più altri tredici) in MO. Questo fatto induce a ritenere che $\mathrm{i}$ tredici mottetti presenti in $\mathrm{MO}$ ma non in $\mathrm{BA}$ siano grosso modo più recenti rispetto agli altri dodici, essendo la compilazione dei due fascicoli finali di MO databile a un'epoca lievemente posteriore rispetto a quella di compilazione di BA. Oltre a questo fatto relativamente ovvio si può osservare come la maggioranza dei mottetti di TO presenti un grado di sofisticazione superiore rispetto a quello delle altre due fonti. Esempi significativi di questa tendenza sono il n. 4, Bele Aelis | Haro Haro I Flos filius, mottetto le cui parti superiori presentano un numero più che triplo di terzine di semibrevi rispetto a BA e MO, e il n. 11, il celebre Aucun ont trouvé| Lonc tans | Annuntiantes, mottetto la cui fisionomia petroniana è accentuata da un cospicuo numero di aggiunte, sia a integrazione di gruppi attestati anche in MO sia a frammentazione di valori colà interi.

A complemento della precedente, la Tabella 4 raccoglie i dati attinenti alle concordanze, non di rado parziali e sovente dotate di varianti significative, con altri manoscritti.

Tabella $4^{183}$

\begin{tabular}{|c|c|c|c|c|}
\hline TO & MANOSCRITTO & $F F$. & PARTI & NOTE \\
\hline 1 & A-VOR, 23 & $1^{\mathrm{r}}$ & 3 & \\
\hline \multirow[t]{2}{*}{2} & A-VOR, 23 & $1^{\mathrm{r}}$ & 3 & \\
\hline & F-Pn, fr. 25566 & $36^{\mathrm{r}}-36^{v}$ & 3 & \\
\hline \multirow[t]{2}{*}{3} & D-Mbs, Clm. 5539 & $78^{r}-79^{v}$ & 3 & $\mathrm{M}$ anche con testo latino \\
\hline & E-BUlh, s/n & $106^{v}-107^{r}$ & 3 & $\mathrm{M}$ con testo latino \\
\hline \multirow[t]{2}{*}{4} & D-DS, 3471 & $\mathrm{III}^{\mathrm{r}}$ & 3 & Tr e M con testo latino \\
\hline & I-Rvat, Reg. lat. 1490 & $115^{\mathrm{v}}-116^{\mathrm{r}}$ & 3 & Tr e M: righi tracciati, ma senza notazione \\
\hline \multirow[t]{4}{*}{5} & E-BUlh, s/n & $120^{\mathrm{r}}-121^{\mathrm{r}}$ & 3 & \\
\hline & GB-Lbl, Add. Ms. 27630 & $50^{v}-51^{r}$ & 3 & \\
\hline & GB-Onc, Ms. 362 & $83^{\mathrm{r}}$ & 3 & \\
\hline & D-TRs, Ms. 322 / 1994 & $214^{v}-215^{r}$ & 3 & T dotato di testo e in tempo doppio \\
\hline \multirow[t]{4}{*}{6} & F-Pn, n.a.fr. 13521 (La Clayette) & $388^{v}$ & 3 & Tr: musica e testo diversi; M: musica diversa \\
\hline & I-Fl, Ms. Pluteus 29.1 & $411^{\mathrm{r}}$ & 2 & $\operatorname{Tr}$ assente; $M$ con testo latino \\
\hline & I-Fl, Ms. Pluteus 29.1 & $163^{v}$ & 2 & Clausula \\
\hline & F-Lm, 316 & $32^{r}-32^{v}$ & 2 & Tr con secondo testo latino; $\mathrm{T}$ assente \\
\hline 8 & F-Dm, 526 & $9^{v}$ & 1 & Solo testo del M \\
\hline \multirow[t]{2}{*}{9} & F-CA, Ms. 410 A & $130^{v}-131^{\mathrm{r}}$ & 2 & Tr assente \\
\hline & GB-Ob, Ms. Douce 308 & $236^{\mathrm{r}}$ & 1 & Solo testo del M \\
\hline \multirow[t]{2}{*}{10} & F-CA, Ms. $410 \mathrm{~A}$ & $129^{\mathrm{r}}$ & 2 & Tr assente \\
\hline & F-Pa, 8521 & $177^{\mathrm{r}}$ & 2 & Solo testo del M \\
\hline \multirow[t]{2}{*}{14} & F-CA, Ms. 410 A & $130^{v}$ & 3 & M con testo latino; parte scambiata con $\operatorname{Tr}$ \\
\hline & I-Tr, Varia 42/2 & $41^{\mathrm{r}}-42^{\mathrm{v}}$ & 3 & $\mathrm{M}$ del n. 28 usato come T \\
\hline \multirow[t]{2}{*}{18} & F-Pn, fr. 12615 (Noailles) & $196^{\mathrm{r}}$ & 1 & Solo M \\
\hline & F-Pn, lat. 15139 (St Victor) & $290^{\mathrm{r}}$ & 2 & Clausula \\
\hline
\end{tabular}

segue

(183) Oltre ai righi relativi ai mottetti nn. 12, 15, 17, 28 e 29, già esclusi nella Tabella 3, nella presente sono esclusi anche quelli relativi ai mottetti nn. 7, 11, 13, 16, 21 e 30, privi di concordanze al difuori delle due raccolte maggiori. Nei casi in cui non è stato possibile compiere verifiche di prima mano, i dati - con esclusione di quelli concernenti le varianti melodiche, soggiacenti a una catalogazione difficile da riportare e superflua nel presente contesto - sono ricavati dagli apparati critici delle edizioni moderne di BA e di MO. 


\begin{tabular}{|c|c|c|c|c|}
\hline \multirow[t]{10}{*}{19} & F-Pn, n.a.fr. 13521 (La Clayette) & $378^{r}-378^{v}$ & 4 & Con Q \\
\hline & D-W, 1206 (olim Helmstad 1099) & $204^{\mathrm{v}}-205^{\mathrm{r}}$ & 3 & \\
\hline & D-W, 1206 (olim Helmstad 1099) & $186^{\mathrm{r}}-186^{\mathrm{v}}$ & 3 & Tr e M con testo latino \\
\hline & D-BAs, Lit. 115 & $49^{\circ}$ & 3 & Tr e M con due nuovi testi latini \\
\hline & D-DS, 3471 & $4 a^{v}$ & 3 & Tr e M con due nuovi testi latini \\
\hline & I-Fl, Ms. Pluteus 29.1 & $157^{v}$ & 2 & Tr e T, clausula \\
\hline & I-Fl, Ms. Pluteus 29.1 & $157^{\mathrm{v}}$ & 2 & M e T, clausula \\
\hline & GB-Lbl, Ms. Add. 30091 & $1^{\mathrm{r}}$ & 2 & Solo M e T; $\mathrm{M}$ con un terzo testo latino \\
\hline & F-BSM & $1^{v}$ & 2 & Solo M e T; $\mathrm{M}$ con un terzo testo latino \\
\hline & D-Mbs, lat. 16444 & $\mathrm{IIa}^{\mathrm{v}}$ & 2 & Solo M e T \\
\hline 20 & GB-Ob, Ms. Douce 139 & $179^{v}$ & 3 & \\
\hline 22 & F-Pn lat., 15139 (St Victor) & $288^{v}-289^{r}$ & 2 & Clausula; $M$ con solo incipit testuale \\
\hline \multirow[t]{2}{*}{23} & F-Pa, 3101 & $5^{\mathrm{r}}$ & 1 & Solo testo del M \\
\hline & GB-Ob, Ms. Douce 308 & $234^{v}$ & 1 & Solo testo del T \\
\hline \multirow[t]{2}{*}{24} & I-Rvat, Reg. lat. 1490 & $114^{v}$ & 3 & \\
\hline & F-CA, Ms. $410 \mathrm{~A}$ & $131^{\mathrm{r}}$ & 2 & Tr assente \\
\hline \multirow[t]{5}{*}{25} & F-Pn, n.a.fr. 13521 (La Clayette) & $375^{v}-376^{r}$ & 3 & \\
\hline & D-BAs, Lit. 115 & $58^{v}-59^{v}$ & 3 & Tr: testo latino \\
\hline & $\mathrm{F}-\mathrm{Pa}, 135$ & $316^{v}$ & 2 & Tr assente \\
\hline & D-EF, Folio 169 & $2 \mathrm{a}^{\mathrm{r}}$ & 3 & $\mathrm{~T}$ diverso \\
\hline & I-Rvat, Reg. lat. 1490 & $115^{\mathrm{r}}-115^{\mathrm{v}}$ & 1 & Solo $\mathrm{Tr}$ \\
\hline \multirow[t]{3}{*}{26} & F-CA, B 1328 & $3(17) \mathrm{a}^{\mathrm{r}}$ & 3 & \\
\hline & I-IV, CXV & $22^{\mathrm{r}}$ & 3 & \\
\hline & I-UDc, 290 & $1^{\mathrm{r}}$ & 3 & \\
\hline \multirow[t]{3}{*}{27} & I-Rvat, Reg. lat. 1543 & $1^{\mathrm{r}}$ & 3 & \\
\hline & F-Pn, n.a.fr. 13521 (La Clayette) & $372^{\mathrm{r}}-372^{v}$ & 4 & Presenza di un Q \\
\hline & F-MO & $390^{\mathrm{r}}-391^{v}$ & 3 & M usato come T, all'ottava inferiore \\
\hline \multirow[t]{4}{*}{31} & F-Pn, n.a.fr. 13521 (La Clayette) & $395^{\mathrm{r}}-395^{v}$ & 3 & \\
\hline & D-Mbs, lat. 16444 & $I V^{v}$ & 3 & Tr e M scambiati, entrambi con testi latini \\
\hline & D-W, 1206 (olim Helmstad 1099) & $219^{v}-219 a$ & 2 & Tr assente \\
\hline & GB-Lbl, Ms. Add. 30091 & $6^{\mathrm{r}}-6^{\mathrm{v}}$ & 2 & Tr assente; $\mathrm{M} \mathrm{e} \mathrm{T}$ con testi latini \\
\hline
\end{tabular}

Le fonti che presentano il numero massimo di concordanze - quattro - sono F-CA Ms. 410 A e F-Pn n.a.fr. 13521 (La Clayette); le altre trasmettono un massimo di due concordanze ciascuna. La quantità e il significato delle note introdotte nell'ultima colonna della tabella amplia notevolmente la nozione di concordanza, estendendola anche ad attestazioni parziali o sensibilmente variate.

Fra le singole composizioni il numero maggiore di (lato sensu) concordanze all'infuori di BA e MO è 9, raggiunto dal mottetto n. 19; seguono, con 5, il mottetto n. 25 e, con 4, i mottetti nn. 5, 6 e 31; e via via tutti gli altri. Il motivo della tradizione manoscritta quantitativamente superiore del n. 19 rispetto a quella degli altri mottetti potrà essere indagato in uno studio futuro; uno studio che potrebbe prendere in considerazione anche un altro argomento importante: quello della presenza di esempi tratti dai mottetti trasmessi da TO e dalle fonti a esso relative nelle opere teoretiche del tempo, dai trattati più celebri a quelli più trascurati, utili anch'essi ai fini di un miglioramento globale nella conoscenza dell'Europa medievale. 